\title{
Advances in Clinical Cardiology 2017: A Summary of Key Clinical Trials
}

\author{
Conor McQuillan · Alastair Gray · Aileen Kearney • Ian B. A. Menown
}

Received: April 18, 2018 / Published online: June 14, 2018

(C) The Author(s) 2018

\section{ABSTRACT}

Introduction: Numerous important cardiology clinical trials have been published or presented at major international meetings during 2017. This paper aims to summarize these trials and place them in clinical context.

Methods: The authors reviewed clinical trials presented at major cardiology conferences during 2017 including the American College of Cardiology, European Association for Percutaneous Cardiovascular Interventions, European Society of Cardiology, European Association for the Study of Diabetes, Transcatheter Cardiovascular Therapeutics, and the American Heart Association. Selection criteria were trials with a broad relevance to the cardiology community and those with potential to change current practice.

Results: A total of 75 key cardiology clinical trials were identified for inclusion. New interventional and structural cardiology data include

Enhanced digital features To view enhanced digital features for this article go to https://doi.org/10.6084/ m9.figshare.6236714.

C. McQuillan · A. Gray · A. Kearney ·

I. B. A. Menown ( $\square)$

Craigavon Cardiac Centre, Southern Trust,

Craigavon, Northern Ireland, UK

e-mail: ian.menown@southerntrust.hscni.net left main bifurcation treatment strategy, multivessel disease management in cardiogenic shock, drug-eluting balloons for in-stent restenosis, instantaneous wave-free physiological assessment, new-generation stents (COMBO, Orsiro), transcatheter aortic valve implantation, and closure devices. New preventative cardiology data include trials of liraglutide, empagliflozin, PCSK9 inhibitors (evolocumab and bococizumab), inclisiran, and anacetrapib. Antiplatelet data include the role of uninterrupted aspirin therapy during non-cardiac surgery and dual antiplatelet therapy following coronary artery bypass grafting. New data are also included from fields of heart failure (levosimendan, spironolactone), atrial fibrillation (apixaban in DC cardioversion), cardiac devices (closed loop stimulation pacing for neuromediated syncope), and electrophysiology (catheter ablation for atrial fibrillation).

Conclusion: This paper presents a summary of key clinical cardiology trials during the past year and should be of practical value to both clinicians and cardiology researchers.

Keywords: Acute coronary syndrome; Anticoagulation; Atrial fibrillation; Bioabsorbable polymer; Cardiology; Coronary revascularization; Heart failure; Lipids; Myocardial infarction; Patent foramen ovale; Transcatheter aortic valve implantation 


\section{INTRODUCTION}

Over the past year, many trials with potential to change clinical practice and influence future clinical guidelines have been presented at international meetings including the American College of Cardiology (ACC), European Association for Percutaneous Cardiovascular Interventions (EuroPCR), European Society of Cardiology (ESC), European Association for the Study of Diabetes (EASD), Transcatheter Cardiovascular Therapeutics (TCT), and the American Heart Association (AHA). In this paper the authors describe new clinical data placed in the context of acute coronary syndrome (ACS), interventional cardiology, heart failure, atrial fibrillation (AF), electrophysiology, and coronary prevention.

\section{METHODS}

The results of major clinical trials in cardiology presented at major conferences in 2017 were reviewed by the authors in addition to a search of PubMed, Medline, Cochrane library, and Embase. Search terms included "acute coronary syndrome," "atrial fibrillation," "coronary prevention," "electrophysiology," "heart failure," and "interventional cardiology." Trials were selected on the basis of relevance to the cardiology community and the potential to change future clinical guidelines or guide further phase 3 research. This article is based on previously conducted studies and does not involve any new studies of human or animal subjects performed by any of the authors.

\section{ADVANCES IN INTERVENTIONAL CARDIOLOGY}

Percutaneous coronary intervention (PCI) is the gold standard for treating the culprit stenosis in patients with ST elevation myocardial infarction (STEMI). However, approximately 50\% of STEMI patients may also have angiographic stenosis in non-culprit arteries and the optimum strategy for managing these non-culprit lesions remains uncertain. The Compare-Acute trial (Fractional Flow Reserve-Guided Multivessel Angioplasty in Myocardial Infarction) randomized 885 patients in 1:2 fashion who had undergone culprit vessel primary PCI to fractional flow reserve (FFR)-guided PCI of nonculprit vessel disease if needed (undertaken in 195 of 295 patients; $83 \%$ immediate, $17 \%$ later during index admission) vs. no further acute PCI $(n=590$ managed electively according to usual care of whom 275 had a blinded FFR of 0.80 or less at time of randomization and 59 underwent non-culprit PCI within the next 45 days) [1]. The primary outcome (the composite of all-cause mortality, nonfatal MI, revascularization, and stroke) occurred less frequently in the FFR-guided complete revascularization group $(7.7 \%$ vs. $20.5 \%, P<0.001)$, mainly driven by the need for repeat revascularization $(6.1 \%$ vs. $17.5 \%, P<0.001)$, of which one-third of cases were for unstable angina. In summary, Compare-Acute confirmed that the figure of approximately $50 \%$ of primary PCI patients having significant non-culprit disease remains true even after objective assessment by FFR, and in keeping with previous studies confirms that complete rather than culprit-only revascularization is preferred (although it does not answer whether the non-culprit PCI should be undertaken acutely or later during early convalescence).

Conventional FFR is undertaken under conditions of maximal hyperemia, requiring use of adenosine or similar drugs which may be poorly tolerated. Instantaneous wave-free ratio (iFR) is a physiological calculation of the resting pressure gradient across a coronary lesion during the portion of diastole when microvascular resistance is low and stable and thus does not require a hyperemic agent. An iFR of 0.89 is considered significant. Previous studies have reported lesion classification by iFR and FFR to be similar but not identical, thus further trials were required to see if these differences were of clinical relevance. The iFR-SWEDEHEART trial (Instantaneous Wave-Free Ratio vs. Fractional Flow Reserve to Guide PCI) randomized 2037 patients to either iFR or FFR-guided PCI [2]. At 12 months, the primary endpoint of death, MI, or revascularization was similar for iFR and FFR $(6.7 \%$ vs. $6.1 \%, P$ non-inferiority $=0.007)$. 
Similarly, the DEFINE FLAIR trial (Use of Instantaneous Wave-Free Ratio or Fractional Flow Reserve in PCI) randomized 2492 patients to either iFR or FFR-guided PCI [3]. At 1 year, the incidence of death, MI, or unplanned revascularization was similar for iFR and FFR (6.8\% vs. $7.0 \%, P$ non-inferiority $<0.001)$. Thus iFR is validated as a reasonable alternative to FFR and may be better tolerated.

PCI of chronic total occlusion (CTO) is typically technically challenging and carries increased procedural time and cost. In the CrossBoss First trial, upfront use of the CrossBoss dedicated CTO crossing catheter (Fig. 1; Boston Scientific) vs. antegrade wire escalation in 246 patients [4] did not significantly shorten crossing time overall (56 vs. $66 \mathrm{~min}, P=0.323$ ) but did shorten crossing in those with in-stent restenosis lesions (41 vs. $66 \mathrm{~min}, P=0.046$ ). While an improvement in angina symptoms may be reported, the value of CTO PCI in terms of hard outcomes has been uncertain.

The first trial to prospectively study such outcomes, the DECISION CTO trial (Drug-Eluting Stent Implantation vs. Optimal Medical Therapy in Patients with Chronic Total Occlusion), randomized 834 patients with stable angina, silent ischemia, or acute coronary syndrome (ACS) in the setting of epicardial CTO in vessels larger than $2.5 \mathrm{~mm}$ to CTO PCI ( $n=417 ; 91 \%$ procedural success) vs. optimal medical therapy $(n=398)$ [5]. At 3-year followup there was no reduction in the primary composite endpoint of death, MI, stroke, or any revascularization with CTO PCI $(19.6 \%$ vs.

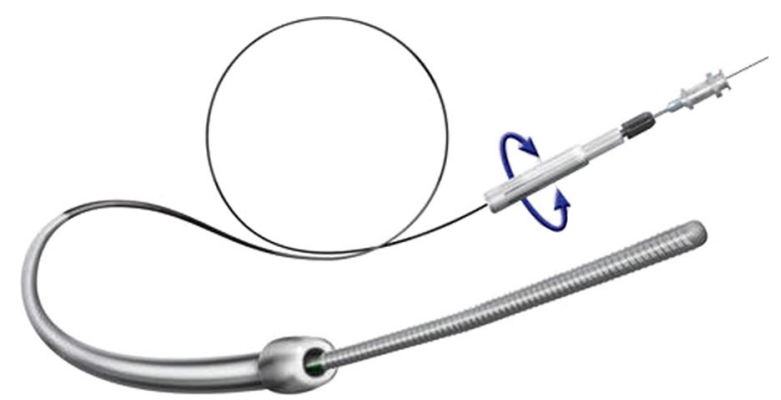

Fig. 1 CrossBoss-dedicated CTO crossing catheter. Image provided courtesy of Boston Scientific. (C)2018 Boston Scientific Corporation or its affiliates. All rights reserved
20.6\%; adjusted HR $0.91 ; P=0.54$ ) nor in individual elements of the composite. Surprisingly CTO PCI was not associated with improvement in angina, exercise tolerance, or quality of life. DECISION CTO [5] was slow to recruit, only enrolled $65 \%$ of the intended patient number, and had a high crossover between groups (20\%) raising the possibility of selection bias and being underpowered. However, the findings do support an initial trial of medical therapy for many patients with CTO.

Another suggested potential benefit of CTO $\mathrm{PCI}$ is recovery of hibernating myocardium. The REVASC CTO trial (A Randomized Multicentre Trial to Evaluate the Utilization of Revascularization or Optimal Medical Therapy for the Treatment of Chronic Total Coronary Occlusions) randomized 205 patients with symptoms and/or positive stress test and EF greater than $25 \%$ to PCI of all CTOs vs. no CTO PCI (although PCI to non-CTO lesions allowed as required). Despite a 99\% procedural success rate (Fig. 2), CTO PCI was not associated with any improvement in regional or global LV function as assessed by MRI [6].

The optimum approach to multivessel CAD revascularization in patients with cardiogenic shock is controversial. Current guidelines suggest complete revascularization is reasonable (Class IIa recommendation) [7]. The CULPRITSHOCK trial (PCI Strategies in Patients with Acute Myocardial Infarction and Cardiogenic Shock) randomized 706 patients acute MI, multivessel disease, and shock to culprit-only PCI (with the option of staged complete revascularization) vs. complete revascularization at the index procedure [8]. The culprit-only PCI group was associated with a lower incidence of the primary endpoint (death or renal replacement therapy at 30 days; $45.9 \%$ vs. $55.4 \%$, $P=0.01)$, decrease in death, reduced contrast use $(190 \mathrm{ml}$ vs. $250 \mathrm{ml}, P<0.001)$, and shorter procedural time. There was no difference in time to hemodynamic stabilization, peak troponin, or duration of inotropic therapy. CULPRIT-SHOCK results challenge current practice and support a conservative culprit-only PCI being preferable.

PCI for distal left main disease is now considered a reasonable alternative to CABG. The 
(a)

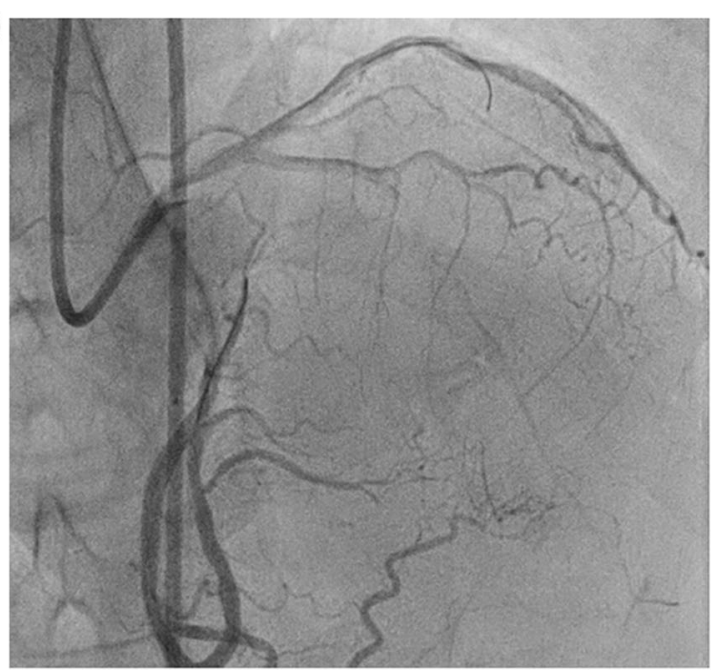

(b)

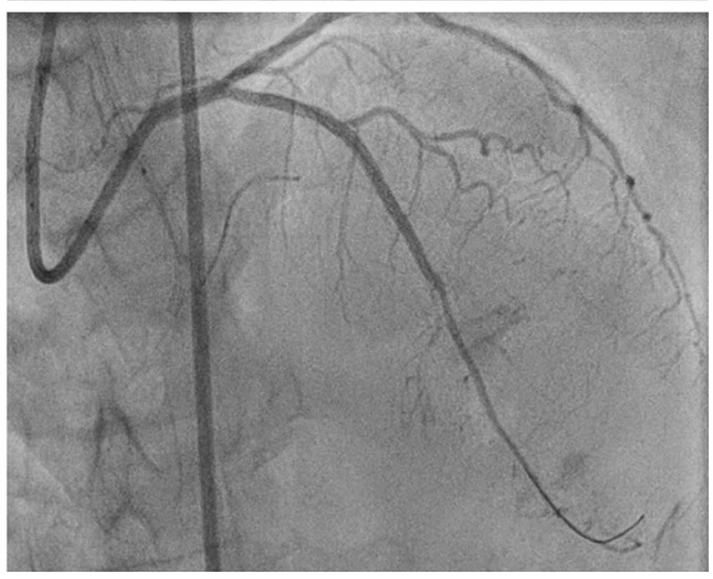

Fig. 2 a LAD CTO with retrograde filling from circumflex septal collaterals. b LAD following recanalization using rCART technique

EXCEL trial (Evaluation of Xience vs. Coronary Artery Bypass Surgery for Effectiveness of Left Main Revascularization), discussed elsewhere [9], reported that PCI vs. CABG was associated with significantly improved measures of quality of life (QOL) as might be expected at 30 days, and remained no worse than CABG at 3 years [10].

However, stent techniques to address bifurcation continue to evolve. The DK-CRUSH V trial (Double Kissing Crush vs. Provisional Stenting for Left Main Distal Bifurcation Lesions) randomized 482 patients with true unprotected distal left main stem disease (Medina $1,1,1$ or $0,1,1)$ to the relatively complex double kissing and double crush (DK-CRUSH) vs. provisional T stenting [11]. DK crush was associated at 1 year with reduced target lesion failure (TLF) $(5.0 \%$ vs. $10.7 \%, P=0.02) \quad \mathrm{MI}$ (0.4\% vs. $2.9 \%, P=0.03)$ and stent thrombosis $(0.4 \%$ vs. $3.3 \%, P=0.02)$ with a trend to reduced clinically driven target lesion revascularization $(3.8 \%$ vs. $7.9 \%, P=0.06)$. Of note, potential confounding factors in the provisional group were the high rate of side branch (SB) dilation (39.7\%), requiring SB stenting in $47.1 \%$ cases, and the undertaking of follow up angiography prior to 12 months which may have increased revascularization rates. DKCRUSH V thus supports a more complex, 2 stent strategy in suitable patients, although the ongoing European Bifurcation Club left main study will add further to this area.

New data evaluating the benefit of PCI vs. medical therapy only for stable angina were reported by the ORBITA (Percutaneous Coronary Intervention in Stable Angina) [12] and FAME 2 (Fractional Flow Reserve vs. Angiography for Multivessel Evaluation) trials [13]. In ORBITA, 200 patients with stable angina, singlevessel disease (Fig. 3; 69\% LAD), and optimized medical therapy (mean number of antianginals 2.8) were randomized to PCI vs. sham procedure [12]. Mean area stenosis was $84.4 \%$ and mean FFR was 0.69. PCI was associated with a significant reduction in ischemic burden (assessed by dobutamine stress echo wall-motion index) and

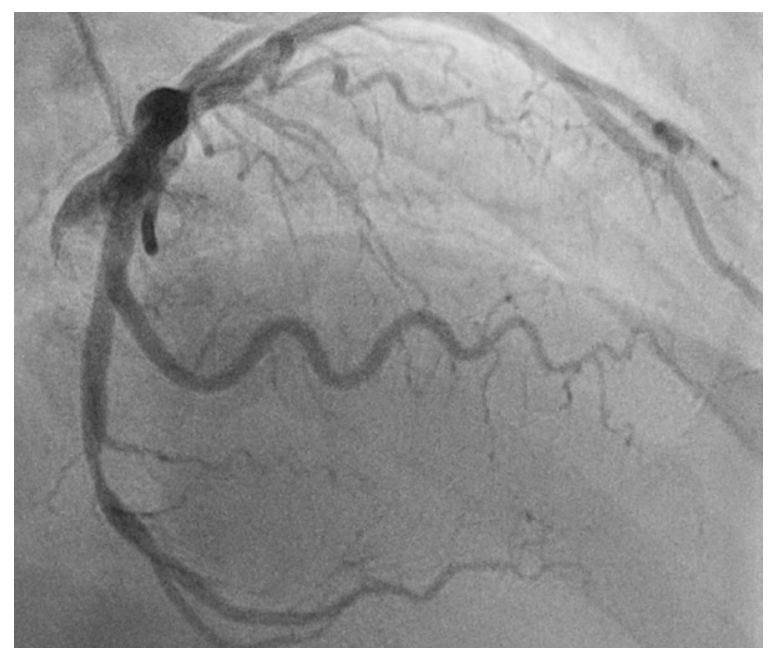

Fig. 3 Single-vessel moderate lesion with FFR value 0.74, typical of that included in the ORBITA trial 
a 16.6-s improvement in exercise time (primary endpoint), although this did not reach the prespecified threshold of $30 \mathrm{~s}(P=0.20)$. Of importance, findings do not apply to multivessel disease in which greater physiological and symptomatic benefit might be expected, nor do they apply to patients with symptoms refractory to medical therapy. In addition, almost onethird of patients undergoing PCI had FFR greater than 0.80 and thus arguably did not require PCI which may have confounded results. The baseline exercise duration was already approximately $8 \mathrm{~min}$ which is good exercise tolerance for older patients and hard to improve further. Nevertheless, ORBITA does suggest that an initial medical trial for stable angina with single-vessel disease is reasonable.

FAME 2 previously randomized 888 patients with single or multivessel disease and FFR 0.80 or less to PCI or medical therapy only. At 3-year follow-up, combined major adverse cardiovascular event (MACE) rates were significantly lower with PCI $(10.1 \%$ vs. $22.0 \%, P<0.001)$ largely driven by lower rates of urgent revascularization $(4.3 \%$ vs. $17.2 \%, P<0.001) \quad[13]$. Although the PCI arm was initially more expensive, by 3 years cumulative totals were similar

$$
(\$ 16,792 \pm 10,139
$$

$\$ 16,737 \pm 13,108 ; P=0.94)$ due to a need for increased PCI later in the medical arm. FFRguided PCI therefore appears an economically favorable strategy being associated with less angina and improved quality of life but similar cost by 3 years.

To date the Xience stent has been considered the gold standard drug-eluting stent (DES). BIOFLOW V (Ultrathin, Bioresorbable Polymer Sirolimus-Eluting Stents vs. Thin, Durable Polymer Everolimus-Eluting Stents in Patients Undergoing Coronary Revascularization) [14] randomized 1334 patients with ACS or stable angina undergoing PCI to the Orsiro stent ( $60 \mu \mathrm{m}$ bioresorbable polymer, sirolimus-eluting; $n=884$ ) vs. Xience (durable polymer, everolimus-eluting; $n=450$ ). Use of ORSIRO was associated with a significant reduction in target lesion failure (TLF) at 30 days (4\% vs. $7 \%$, $P=0.03)$ and at 12 months $(6 \%$ vs. $10 \%$, $P=0.04)$ driven by reduced target vessel MI (5\% vs. $8 \%, P=0.02$ ) including reduced in-hospital peri-procedural MI (3.9\% vs. $6.7 \%, P=0.029)$, although the exact mechanism for this is unclear and the TLF rate in the Xience group was higher than in previous studies. Nevertheless, BIOFLOW V suggests that the third-generation stents with ultrathin struts, such as Orsiro, may now represent a new gold standard for DES outcomes.

Data regarding first-generation fully bioresorbable vascular scaffolds (BVS) has continued to be disappointing (Fig. 4). While 1-year data from the ABSORB III trial (Clinical Outcomes with Everolimus-Eluting Bioresorbable

(a)

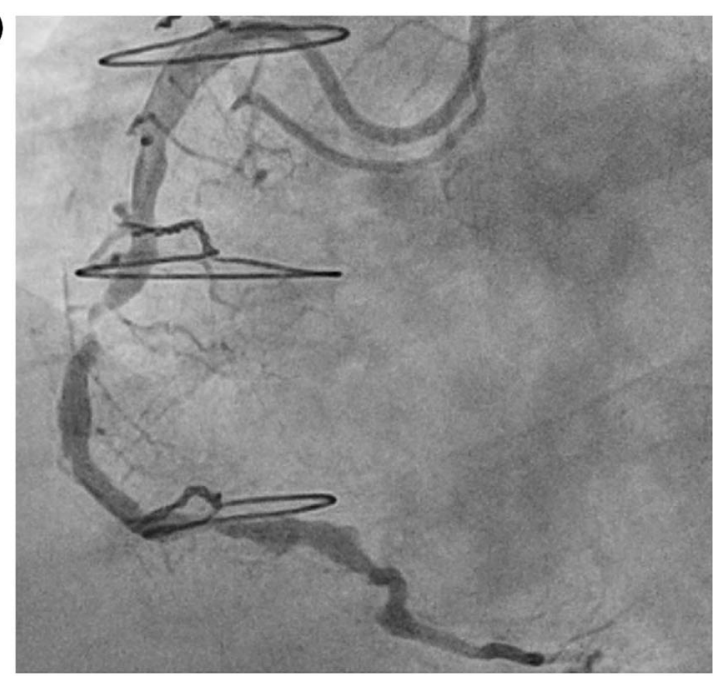

(b)

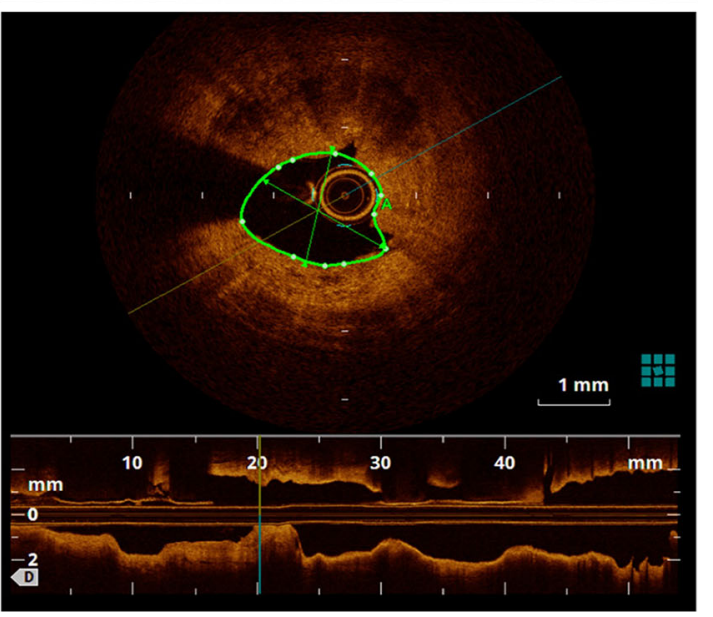

Fig. 4 a Target lesion failure with severe in-stent restenosis 3 years following revascularization with BVS. b Corresponding optical coherence tomography image with reduced minimal luminal area and intimal hyperplasia 
Coronary Scaffolds) reported similar TLF outcomes for BVS and DES, at 3 years, use of BVS was associated with a trend to increased TLF (13.4\% vs. $10.4 \%, P=0.06)$, increased target vessel MI $(8.6 \%$ vs. $5.9 \%, P=0.03)$, and increased stent thrombosis $(2.3 \%$ vs. $0.7 \%$, $P=0.01)$ especially in small vessels of less than $2.25 \mathrm{~mm}$ [15]. The Absorb stent has subsequently been withdrawn.

While use of DES vs. bare metal stents has reduced incidence of in-stent restenosis (ISR), when ISR occurs, optimum management remains uncertain. The DARE trial (Drug-Eluting Balloon for In-Stent Restenosis) randomized 278 patients with ISR (56\% DES-ISR and 44\% BMS-ISR) to the SeQuent Please drug (paclitaxel)-eluting balloon (DEB) $(n=137)$ vs. Xience DES $(n=141)$ [16]. Use of DEB was associated with a smaller immediate minimal lumen diameter (MLD) $(1.72 \pm 0.35 \mathrm{~mm}$ vs. $1.84 \pm 0.46 \mathrm{~mm}, P=0.018$ ) but no significant difference in MLD at 6 months, a reduction insegment late loss $(0.17 \mathrm{~mm}$ vs. $0.45 \mathrm{~mm}$, $P<0.001)$, and no difference in need for further target vessel revascularization $(8.8 \%$ vs. $7.1 \%$, $P=0.65)$. Since it avoids a further layer of metal, DEB may be a useful option for treatment of ISR particularly if overriding a side branch.

While the optimal duration of dual antiplatelet therapy (DAPT) following acute coronary syndromes (ACS) remains a matter of debate, in patients at high bleeding risk, a shorter DAPT course is desirable. Quick healing DES may facilitate this. The REDUCE trial (Short Term Dual Antiplatelet Therapy in Patients with ACS Treated with the COMBO Dual Therapy Stent) randomized 1496 patients with ACS to 3 vs. 12 months DAPT following implantation of the COMBO stent (bioabsorbable polymer-based metallic abluminal sirolimus DES and a luminal CD34+ antibody coating) [17]. Use of a shortened DAPT course was not associated with a difference in the primary endpoint (death, MI, moderate/major bleeding- $8.2 \%$ vs. $8.4 \%$ ). There was a numerical increase in incidence of stent thrombosis $(1.1 \%$ vs. $0.4 \%, P=0.13)$ and mortality $(1.9 \%$ vs. $0.8 \%)$ but the study was not powered to test individual endpoints. In keeping with ESC guidelines, a shorter course of DAPT may be considered for selected patients at high bleeding risk.
PCI operators are exposed to significant levels of radiation. The RECAP trial (Efficacy of the RADPAD Protection Drape in Reducing Operators Radiation Exposure in the Catheterization Laboratory) [18] randomized 766 consecutive coronary procedures in 1:1:1 fashion to RADPAD (a sterile, disposable, lead-free shield placed on the patient; $n=255$ ), NOPAD (usual care; $n=255$ ), or SHAMPAD (a sham non-protective shield $=256$ ). Use of RADPAD was associated with a $20 \%$ reduction in radiation exposure vs. NOPAD and a $44 \%$ reduction vs. SHAMPAD (which may reflect a false sense of security and other suboptimal practice when a PAD is in place). RECAP supports use of RADPAD in all labs, and may be of particular value for younger interventionists likely to be exposed to a high lifetime cumulative radiation dose.

Cardiac surgical patients often require blood transfusion in the postoperative period with many centers transfusing liberally. The TRiCS (Transfusion Requirement in Cardiac Surgery) trial evaluated a restrictive (transfusion if hemoglobin $<7.5 \mathrm{~g} / \mathrm{dl}$ ) vs. liberal (transfusion if hemoglobin $<9.5 \mathrm{~g} / \mathrm{dl}$ ) red cell transfusion strategy in 5092 adults at moderate to high risk undergoing cardiac surgery (CABG 26.1\%, CABG with other procedure $27.7 \%$, non-CABG surgery 46.2\%) [19]. Transfusion (median 2 units) was given to $52.3 \%$ patients in the restrictive group compared to $72.6 \%$ in the liberal group with no change in the primary composite endpoint of death, MI, stroke, or renal failure, suggesting that a conservative approach to blood transfusion appears safe and may be preferable in this high risk population.

Contrast-associated acute kidney injury (AKI) following coronary angiography is associated with increased morbidity and mortality but attempts to reduce this with medical therapy have been disappointing. The PRESERVE trial (Prevention of Serious Adverse Events Following Angiography) randomized 4993 patients undergoing coronary procedures (mean contrast volume $85 \mathrm{ml})$ and at risk of AKI $(80.9 \%$ had diabetes mellitus, median creatinine clearance $50.2 \mathrm{ml} / \mathrm{min}$ ) in a 2-by-2 factorial design to $1.26 \%$ sodium bicarbonate vs. $0.9 \%$ sodium chloride and $\mathrm{N}$-acetylcysteine vs. placebo [20]. 
In keeping with previous trials, neither sodium bicarbonate nor $N$-acetylcysteine was associated with a reduction in the primary endpoint (death, dialysis, or persistent increase in creatinine at 90 days) nor the rate of contrast-associated AKI.

\section{ADVANCES IN STRUCTURAL CARDIOLOGY}

Transcatheter aortic valve implantation (TAVI) is superior to medical therapy for treatment of high risk patients with severe aortic stenosis (AS) deemed unsuitable for surgical aortic valve replacement (SAVR); class $1 \mathrm{~A}$ recommendation [21]. For patients at intermediate risk with mean STS (Society of Thoracic Surgeons) score of 4-8, the PARTNER 2 (Placement of Aortic Transcatheter Valves) study suggested that TAVI may be a reasonable alternative to SAVR [9], but further studies were required. Further support now comes from the SURTAVI trial (Surgical or Transcatheter Aortic Valve Replacement in Intermediate Risk Patients) which randomized 1660 patients with symptomatic severe AS and mean STS score $4.5 \pm 1.6 \%$ to TAVI $(n=864)$ or SAVR $(n=796)$ [22]. At 24 months, there was no difference in the primary composite endpoint of all-cause death or disabling stroke $(12.6 \%$ vs. $14.0 \%$, posterior probability of non-inferiority $>0.999)$. TAVI was associated with less residual mean aortic valve gradient (7.8 vs. $11.8 \mathrm{mmHg})$, AF (12.9\% vs. $43.4 \%)$, acute kidney injury (1.1\% vs. $4.4 \%)$, or early stroke $(3.3 \%$ vs. $5.4 \% ; P=0.031$ at 30 days, 6.3 vs. $8.0 \%$; $P=0.143$ at 2 years) but greater moderate or severe residual para-valvular regurgitation $(5.3 \%$ vs. $0.6 \%)$, vascular complication $(6.0 \%$ vs. $1.1 \%)$, and need for pacemaker implantation $(25.9 \%$ vs. $6.6 \%)$. It is likely these SURTAVI data will lead to an enhanced guideline recommendation for use of TAVI in intermediate risk patients.

Although TAVI incurs higher index procedural cost, an analysis from PARTNER 2A and SAPIEN3 trials of patients undergoing TAVI or SAVR suggested that TAVI was cost-effective at 2-year clinical follow-up and was predicted to become the economically dominant strategy by lifetime horizon [23] at least in an Americanbased healthcare system.

Conduction disturbance is the most common complication following TAVI with small studies providing conflicting evidence on the significance of left bundle branch block (LBBB) post TAVI, but some of which suggest a higher incidence of sudden cardiac death. The MARE trial (Ambulatory ECG Monitoring with an Implantable Loop Recorder in Patients with New Onset LBBB Following TAVR) implanted loop recorders in 103 patients with persistent LBBB following TAVI [24]. At 12-month followup, $10 \%$ patients had brady-arrhythmias or high grade AV block requiring pacemaker, 17\% patients had new $\mathrm{AF}$, and $14 \%$ had new episodes of ventricular tachycardia (with similar incidence whether using self-expanding or balloon expanding valves). The high rate of subsequent arrhythmias in these often asymptomatic patients supports consideration of ILR as extended monitoring in this cohort.

Leaflet thrombosis with TAVI may be more common than first anticipated and associated with important clinical risk. The Manufacturer and User Facility Device Experience (MAUDE) database reported 30 cases of structural valve dysfunction due to leaflet thrombosis between January 2012 and October 2015 from a total of 5691 TAVI-related adverse events. Of these 30, 18 (60\%) presented during the 1st year following TAVI as either aortic stenosis (53.3\%), aortic regurgitation (23.3\%), or both (13.3\%) [25]. Treatment was attempted by escalation of antiplatelet/anticoagulant (30\%), valve-in-valve TAVI (16.7), or surgery (46.7\%) but still resulted in adverse outcomes including stroke (10\%), cardiogenic shock (6.7\%), or death (30\%). Studies are thus ongoing to evaluate methods of reducing TAVI leaflet thrombosis.

Both TAVI and SAVR may be associated with postoperative embolic stroke. The SENTINEL trial (Cerebral Protecting in Transcatheter Aortic Valve Replacement) previously showed that use of a transcatheter embolic protection device (Fig. 5) captured embolic debris in 99\% of patients undergoing TAVI but was only associated with a small, non-significant reduction in strokes at 30 days $(5.6 \%$ vs. 9.1\%) $[9,26]$. A similar strategy has since been tested in 383 
(a)

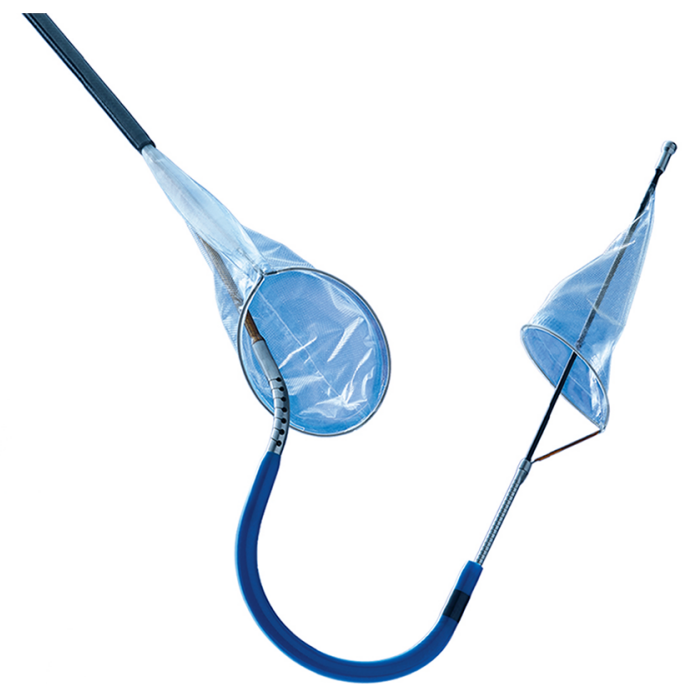

(b)

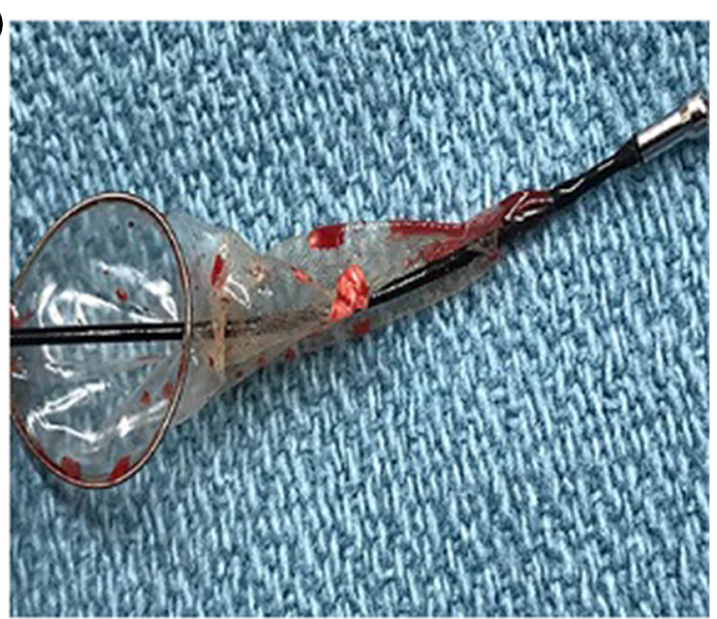

(c)

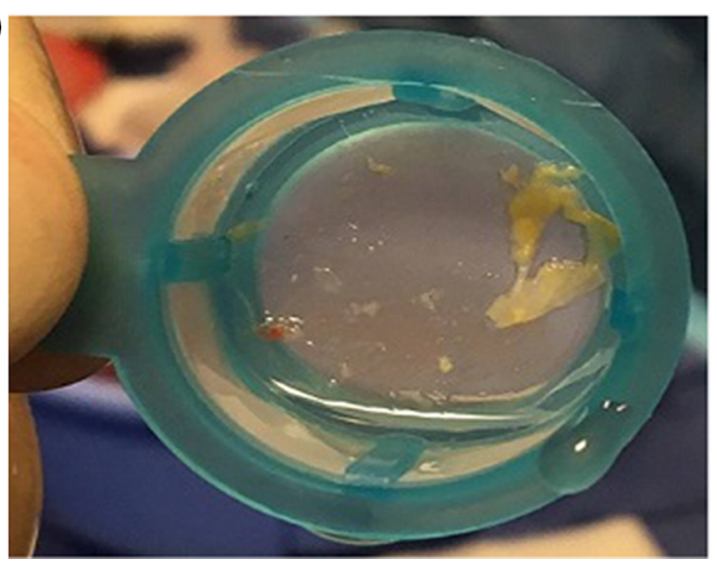

Fig. 5 a SENTINEL cerebral protection device. b, c Debris caught by cerebral protection device during TAVR. Images reproduced with permission from Claret Medical patients undergoing SAVR using the CardioGuard and Embol-X cerebral embolic protection devices [27]. The Embol-X device collected debris in 99\% patients and the CardioGuard in $68 \%$ of patients. There was no significant clinical or radiographic reduction in stroke, but both devices were associated with a significant reduction in delirium. Further trials with embolic protection devices in TAVR and SAVR settings are planned.

Percutaneous mitral valve devices are developing rapidly and moving from preclinical to clinical studies. Given the complexity of the mitral valve, different approaches are needed depending on the pathology of valvular dysfunction. In the TRACER (Mitral TransApical Cordal Echo-Guided Repair) trial, 30 patients with severe degenerative MR due to posterior leaflet prolapse underwent percutaneous repair using the Harpoon transventricular beating heart device which utilizes polytetrafluoroethylene cords [28]. Technical success was achieved in 28/30 cases with MR being reduced to none/trace in $86 \%$ or mild in $14 \%$ and zero serious complications including stroke, death, renal failure, or need for permanent pacemaker implantations. Results were sustained to 6 months with $85 \%$ having none/mild MR, 8\% moderate MR, and 8\% severe MR. Large studies are planned.

Stroke remains a concerning complication not only with SAVR but also with general cardiac surgery. This may be partly related to postoperative AF which can occur in 30-67\% patients in the year following cardiac surgery. $\mathrm{AF}$ is associated with thrombus formation in the left atrial appendage (LAA). However, conflicting results have been reported from observational studies of routine prophylactic LAA closure at the time of cardiac surgery. The Left Atrial Appendage Closure by Surgery (LAACS) study is the first randomized study in which 187 patients were assigned to LAA closure (by ligation with silk suture; $n=101$ ) or no closure $(n=86)$ at the time of CABG [29]. Over a mean follow-up of 3.6 years, LAA closure was associated with a $70 \%$ reduction in the primary composite endpoint of ischemic stroke or cerebral infarct on MRI (5\% vs. $16 \%, P=0.02)$ with a trend to reduction in clinically significant stroke $(P=0.07)$. Conclusions were limited by the small trial size and single-center setting but the findings encourage further study. The 
optimum method for surgical LAA closure also requires further study-endocardial suturing was considered by the LAACS investigators to have lower bleeding risk but may leave a remnant of LAA in more cases than clipping or stapling.

Trials of percutaneous LAA closure (Fig. 6) initially reported mixed results: PREVAIL (Prospective Randomized Evaluation of the Watchman Left Atrial Appendge Closure Device in Patients with AF vs. Long-Term Warfarin Therapy) failed to show statistical non-inferiority for the composite primary endpoint of stroke, systemic embolism, or death (thought due to an expectedly low stroke rate in the warfarin arm) and PROTECT AF (Watchman Left Atrial Appendage System for Embolic Protection in Patients with AF) reported a similar incidence of stroke with LAA closure and warfarin but a high rate of device complications. Long-term results are now available [30]. At 5-year follow-up, PREVAIL showed non-inferiority for the co-primary endpoint of post-procedure ischemic stroke/systemic embolism (although not the co-primary endpoint of stroke/systemic embolism or cardiovascular/ unexplained death). In a meta-analysis of PREVAIL and PROTECT ( $n=1114$ patients) the composite endpoint was non-inferior with numerically higher ischemic stroke/systemic embolism $(P=0.08)$ but lower rates of hemorrhagic stroke, disabling/fatal stroke, and allcause death (all $P<0.05$ ). Comparative data of LAA closure vs. direct oral anticoagulants (now a class IA recommendation for AF) are needed but the meta-analysis strongly supports consideration of LAA closure in patients with $\mathrm{AF}$ but unable to take oral anticoagulation.

Previous trials of patent foramen ovale (PFO) closure in patients with cryptogenic stroke have been confounded by small sample sizes and other multifactorial factors. The REDUCE trial (Patent Foramen Ovale Closure or Antiplatelet Therapy for Cryptogenic Stroke) investigators randomized 664 patients $(81 \%$ with moderate to large intra-atrial shunts) to PFO closure (with the Gore Helex Septal Occluder or the Gore Cardioform Septal Occluder) plus antiplatelet therapy vs. antiplatelet therapy alone [31]. During a median follow-up of 3.2 years, PFO closure was associated reduction in clinical stroke $(1.4 \%$ vs. $5.4 \%, P=0.002)$ and radiological new brain infarction $(5.7 \%$ vs. $11.3 \%$, $P=0.04)$, but no difference in silent brain infarction and higher incidence of AF (6.6\% vs. $1.4 \%$ ). The CLOSE trial (Patent Foramen Ovale Closure or Anticoagulation vs. Antiplatelets After Stroke) randomized 663 patients (with

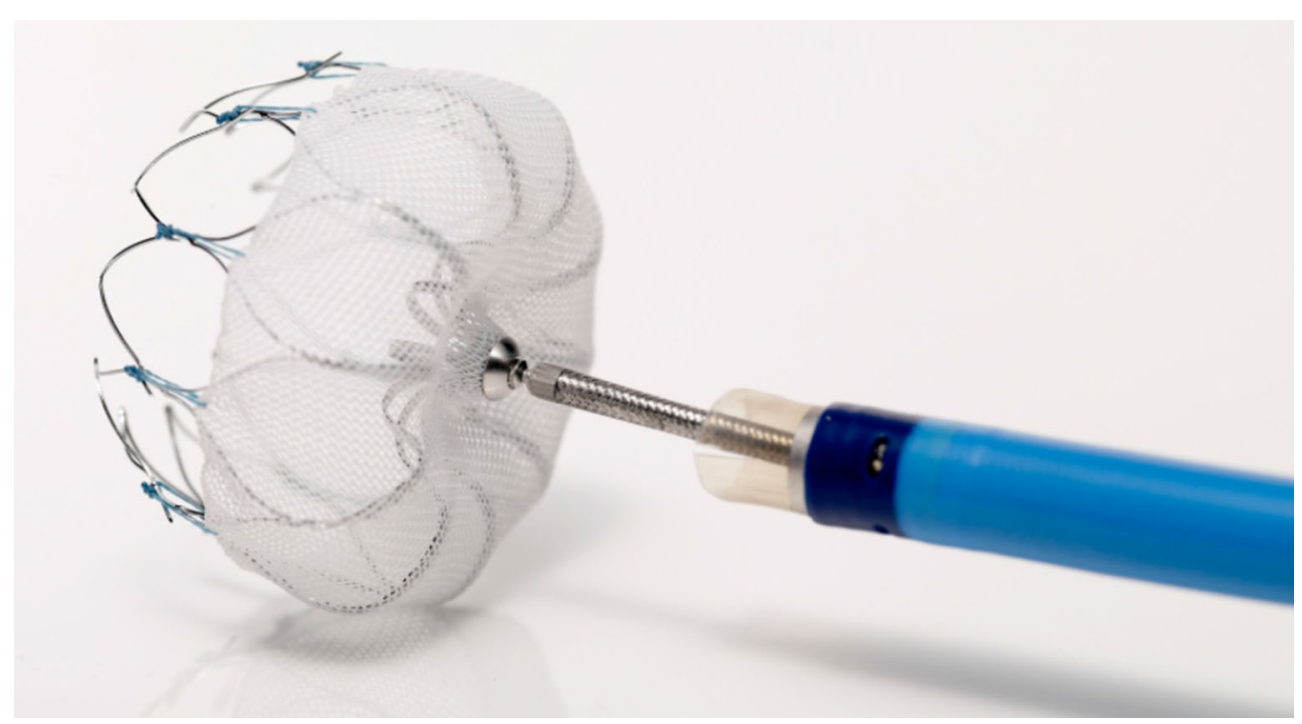

Fig. 6 Watchman left atrial appendage closure device mounted on delivery catheter. Image provided courtesy of Boston Scientific. (C) 2018 Boston Scientific Corporation or its affiliates. All rights reserved 
atrial septal aneurysm or large shunt) to PFO closure (any approved device) plus antiplatelet therapy vs. antiplatelet therapy alone vs. anticoagulation alone [32]. During a mean followup of 5.3 years, PFO closure was associated with reduction in clinical stroke vs. antiplatelets alone ( 0 vs. 14 patients, $P<0.001$ ). New onset AF following PFO closure was observed in $4.6 \%$ patients. There was a trend to benefit of anticoagulation over antiplatelet therapy alone which did not reach statistical significance. Along with extended follow-up from the RESPECT trial (Long-term Outcomes of Patent Foramen Ovale Closure or Medical Therapy After Stroke), presented in 2016 [9], which reported a reduction in recurrent ischemic stroke with PFO closure vs. medical therapy (hazard ration $0.55, P=0.046$ ), REDUCE and CLOSE support updating guidelines for change in guidelines management of patients following cryptogenic stroke.

Coinciding with a growing evidence base supporting PFO closure are new technologies including the NobelStitch which delivers separate polypropylene KwiKnot sutures to the primum and secundum septae via 12-French access under local anesthetic. Average procedure time was $65 \mathrm{~min}$ in the 78 patients included in this trial where no residual shunt was observed in $79 \%$ cases with no procedural related complications, arrhythmias, or recurrent cerebral embolic events at 6 months [33]. Since a permanent device is not left behind, this may negate the need for long-term antiplatelet or anticoagulation.

\section{ADVANCES IN CARDIOVASCULAR PREVENTION}

The STAMPEDE trial (Surgical Therapy And Medications Potentially Eradicate Diabetes Efficiently) trial, which showed that bariatric surgery improved rates of achieving normoglycemia vs. medical therapy after 5 years, also suggested that blood pressure (BP) control might be improved [34]. The GATEWAY (Gastric Bypass to Treat Obese Patients with Steady Hypertension) trial prospectively randomized 100 patients with elevated body mass index and hypertension to gastric bypass plus medical therapy vs. medical therapy alone [35]. At 12 months, gastric bypass was associated with reduction in need of medical therapy in $51 \%$ and $45.8 \%$ of patients, based on office and $24 \mathrm{~h}$ BP monitoring, respectively, achieving remission of hypertension, whereas no patient randomized to medical therapy alone was free of antihypertensive drugs.

Maintaining BP control remains a challenge. The BP TITRE (Time at Blood Pressure Target and the Risk of Cardiovascular Diseases and Mortality) study incorporated longitudinal information on BP control and its impact on $\mathrm{CV}$ outcomes in almost 170,000 individuals with newly identified hypertension but no prior $\mathrm{CV}$ disease [36]. Length of time within target BP range predicted treatment benefit. Those maintaining BP targets 9-11.9 months per year vs. 3-5.9 months per year showed a 30\% reduction in CV death, MI, and stroke. Those who failed to meet target $\mathrm{BP}$ range in any months per year had the greatest risk of $\mathrm{CV}$ death, MI, and stroke $(n=108$; odds ratio 4.51 , 95\% CI 3.64-5.52). BP TITRE highlights the importance of patient follow-up in maintaining control even if initial targets have been achieved.

The 2015 SPRINT (Systolic Blood Pressure Intervention Trial) trial reported that intensive systolic (SBP) control $(<120 \mathrm{mmHg})$ vs. standard control $(<140 \mathrm{mmHg})$ resulted in $\mathrm{CV}$ benefit [37]. However, questions were raised about the comparability of BP readings with other studies since in SPRINT, after 5 min rest, BP measurement was undertaken with an automated device and staff may or may not have been in attendance (4082 always alone, 2247 never alone, 1746 alone during rest, 570 alone during BP measurement). At AHA 2017 [38] post hoc analysis reported no significant difference in BP levels or in CV outcomes whether assessment was attended or unattended and thus it is reasonable to extrapolate SPRINT findings to both attended or unattended BP measurement settings.

On the basis of SPRINT and other trials, updated ACC/AHA guidelines have lowered BP definitions [39] with elevated BP defined as $120-129 /<80 \mathrm{mmHg} \quad$ while stage 1 
hypertension is defined as 130-139/80-$89 \mathrm{mmHg}$. These reduced thresholds help guide earlier non-pharmacological intervention. The benefit of combining the DASH (Dietary Approaches to Stop Hypertension) diet (high in fruits/vegetables/low-fat dairy; low in saturated fat/cholesterol) [40] and a reduced sodium diet [41] was evaluated among 412 patients with hypertension in the DASH-Sodium trial [42]. Those randomized to the combination diet showed greater reduction in SBP compared with DASH or low sodium diets alone, with the greatest benefits at highest baseline systolic BP levels $(P$ for trend $=0.004)$. For patients with baseline SBP $\geq 150 \mathrm{mmHg}$, use of combination diet vs. high sodium/control diet was associated with a $-20.8 \mathrm{mmHg}$ reduction in SBP. This highlights the value of dietary intervention in this high risk group.

The role of catheter-based renal denervation in the management of hypertension has been uncertain. SYMPLICITY HTN3 did not show significant BP benefit vs. a sham procedure, but factors such as limited extent of denervation, inexperienced operators, nonadherence to medication, and patient selection may have influenced results $[43,44]$. The SPYRAL HTNOFF MED trial evaluated the effect of renal denervation in patients with mild-moderate hypertension $\quad$ (office $\quad \mathrm{SBP} \geq 150$ to $<180 \mathrm{mmHg}$, office diastolic BP (DBP) $\geq 90 \mathrm{mmHg}$ and mean $24 \mathrm{~h} \mathrm{SBP} \geq 140$ to $<170 \mathrm{mmHg}$ ) not on antihypertensives [45]. This single blind, sham-controlled trial randomized 80 patients to renal denervation ( $n=38$; mean 44 ablations per patient; 18 main artery, 26 branch artery) or sham control $(n=42)$. After 3-month follow-up the renal denervation group had a modest but statistically significant reduction in office and ambulatory $\mathrm{BP}(24 \mathrm{~h}$ SBP $-5.5 \mathrm{mmHg}, P=0.0031$; $24 \mathrm{~h}$ DBP $-4.8 \mathrm{mmHg}, P<0.001$; office SBP - $10 \mathrm{mmHg}, \quad P<0.004 ; \quad$ office $\quad$ DBP $-5.3 \mathrm{mmHg}, \quad P=0.0002$ ) whereas the sham group showed no significant changes in BP. Although a small trial, SPYRAL HTN-OFF MED finally provides proof of concept for renal denervation (as undertaken by latest protocols) and may guide future larger trials.
Significant reduction in CV death, MI, or stroke has been reported with daily administration of the glucagon-like peptide 1 (GLP-1) receptor agonist liraglutide in patients with type 2 diabetes mellitus [46]. The Exenatide Study of Cardiovascular Event Lowering Trial (EXSCEL) randomized 14,752 patients with type 2 diabetes to once weekly exenatide vs. placebo [47]. Exenatide was only associated with a non-significant reduction in the primary outcome of $\mathrm{CV}$ death, MI, or stroke vs. placebo (11.4\% vs. $12.2 \% P=0.06$ ) but did lead a $14 \%$ reduction in the secondary endpoint of all-cause mortality vs. placebo $(6.9 \%$ vs. $7.9 \%$, HR $0.86,95 \%$ CI 0.77-0.98)—an observation which appeared consistent across patient subgroups. However, as the primary endpoint was not met, the mortality data can only be considered hypothesis-generating.

Patients with co-existing type 2 diabetes mellitus and peripheral artery disease (PAD) are at heightened risk of CV complications. Empagliflozin, a sodium-glucose cotransporter 2 (SGLT2) inhibitor, has previously been shown to reduce $\mathrm{CV}$ death, all-cause mortality, and hospitalization for heart failure by $38 \%, 32 \%$, and $35 \%$, respectively, vs. placebo [48]. New data from the PAD subgroup of EMPA-REG OUTCOME ( $n=1461$ patients; $20.8 \%$ ) reported even greater treatment with empaglifozin vs. placebo including reduction of $\mathrm{CV}$ death by $43 \%$ (HR 0.57, 95\% CI 0.37-0.88), all-cause mortality by $38 \%$ (HR $0.62,95 \%$ CI $0.44-0.88$ ), and hospitalization for heart failure by $44 \%$ (HR 0.56, 95\% CI 0.35-0.92). There was no difference in rates of lower limb amputations [49].

CANVAS (Canagliflozin for Primary and Secondary Prevention of Cardiovascular Events in Type 2 Diabetes) randomized 10,142 patients with type 2 diabetes to the SGLT2 inhibitor canagliflozin vs. placebo [50]. At a median follow-up of 188 weeks canagliflozin was associated with a $14 \%$ reduction in the primary outcome of CV death, MI, or stroke (26.9 vs. 31.5 per 1000 patient-years, HR 0.86; 95\% CI 0.75-0.97). In contrast to EMPA-REG OUTCOME, the numerical reductions in $\mathrm{CV}$ death and all-cause death were not significant, although the 33\% reduction in hospitalization for heart failure and $27 \%$ reduction in 
progression of albuminuria were significant. Unexpectedly, canagliflozin was associated with increased risk of amputation, primarily toe or metatarsal vs. placebo (6.3 vs. 3.4 cases per 1000 patient-years, HR 1.97) [49]. In summary, SGLT2 inhibitors canagliflozin and empagliflozin have favorable $\mathrm{CV}$ benefit in patients with type 2 diabetes, although empagliflozin may be preferable in those with concurrent PAD [51].

Proprotein convertase subtilisin/kexin type 9 (PCSK9) inhibitors when added to statin therapy are associated with marked further reductions in low density lipoprotein cholesterol (LDL-C), but to date it has not been known if this translates into CV benefit. FOURIER (Further Cardiovascular Outcomes Research with PCSK9 Inhibition in Subjects with Elevated Risk) randomized 27,564 patients with prior MI, stroke, or PAD and already receiving statin to evolocumab, a fully human monoclonal antibody (140 mg every 2 weeks or $420 \mathrm{mg}$ monthly) vs. placebo [52]. After a median follow-up of 26 months, evolocumab vs. placebo was associated with a $15 \%$ reduction in the composite of CV death, MI, stroke, coronary revascularization, or unstable angina hospitalization $(9.8 \%$ vs. $11.3 \%$; $P<0.001)$. Benefit was similar for all baseline LDL-C levels including the patient subgroup who dropped to $0.6 \mathrm{mmol} / \mathrm{l}$ after treatment with no evidence of a "J curve phenomenon" (i.e., no level below which benefit stopped being observed). The key secondary endpoint, CV death, MI, or stroke was reduced by $20 \%(P<0.001)$, driven by a $27 \%$ reduction in MI $(P<0.001), 21 \%$ reduction in stroke $(P=0.01)$, and $22 \%$ reduction in coronary revascularization $(P<0.001)$. There was no reduction observed for $\mathrm{CV}$ death, although given the baseline profile, this might require several years' follow-up to emerge.

In the FOURIER subgroup with PAD $(n=3642)$ [53] evolocumab reduced the primary endpoint by $21 \%$ (HR $0.79 ; 95 \%$ CI 0.66-0.94) and there was a significant reduction in the incidence of major adverse limb events $(0.27 \%$ vs. $0.45 \% P=0.0093)$. In the FOURIER subgroup with prior MI [54] $(n=22,351)$, the primary endpoint treatment benefit of evolocumab was most marked in those with more
Table 1 Reduction in primary endpoint outcome with evolocumab in patients with previous MI

\begin{tabular}{llr}
\hline $\begin{array}{l}\text { High risk } \\
\text { feature }\end{array}$ & $\begin{array}{l}\text { HR }(95 \% \text { CI }) \text { for primary } \\
\text { endpoint reduction with } \\
\text { evolocumab }\end{array}$ & $\boldsymbol{P}$ value \\
\hline$<2$ years from & $0.76(0.64-0.89)$ & $<0.001$ \\
$\begin{array}{l}\text { most recent MI } \\
\geq 2 \text { prior MIs }\end{array}$ & $0.79(0.67-0.94)$ & 0.006 \\
$\begin{array}{l}\text { Multivessel } \\
\text { coronary artery } \\
\text { disease }\end{array}$ & $0.70(0.58-0.84)$ & $<0.001$ \\
\hline
\end{tabular}

recent ( $<2$ years) MI, more than two prior MIs, or multivessel CAD (Table 1). Given the current cost of PCSK9 inhibitors, these subgroup data may help target treatment to patients likely to derive greatest benefit.

Whilst the ability to achieve marked reduction in LDL-C is welcomed, there has been caution surrounding the possible effects on cognitive function [55]. EBBINGHAUS (Evaluating PCSK9 Binding Antibody Influence on Cognitive Health in High Cardiovascular Risk Subjects) assessed a subgroup of 1204 patients from the FOURIER trial [56] patients who received either evolocumab or placebo in addition to using the Cambridge Neuropsychological Test Automated Battery. The primary endpoint was the score on the spatial working memory strategy index of executive function and secondary endpoints were scores of working memory, episodic memory, and psychomotor speed. There was no significant difference in cognitive function between evolocumab and placebo over a median follow-up of 19 months even in patients who achieved very low levels of LDL cholesterol. Whilst the results are encouraging the follow-up period was short and longer studies are desirable given that treatment of hypercholesterolemia typically spans over decades.

Bococizumab is an inhibitor of PCSK9 which, unlike evolocumab, is a humanized monoclonal antibody in which approximately $3 \%$ of the murine sequence remains in the antigen- 
binding complementarity-determining region. The SPIRE program (Studies of PCSK9 Inhibition and the Reduction of Vascular Events) evaluating bococizumab $150 \mathrm{mg}$ every fortnight in patients with CV disease [57] was stopped early and the development of bococizumab discontinued because of the development of antidrug antibodies in almost half of patients. Before being prematurely stopped, 16,817 lower risk patients with LDL-C $\geq 70 \mathrm{mg} / \mathrm{dl}$ and 10,621 higher risk patients with LDL-C $\geq 100 \mathrm{mg} / \mathrm{dl}$ had been recruited in SPIRE-1 and SPIRE-2,respectively. After a median follow-up of 10 months bococizumab did not show a significant reduction of primary endpoint (CV death, $\mathrm{MI}$, stroke, or hospitalization for unstable angina requiring urgent revascularization) in lower risk patients but it did show a reduction in higher risk patients (179 vs. 224 , HR $0.79,95 \%$ CI $0.65-0.97, P=0.02)$. Overall, the SPIRE data are in keeping with the FOURNIER results, although they highlight the value of using human monoclonal antibodies particularly for chronic repeated administration treatments.

Small interfering RNAs (siRNAs) provide a novel approach to reduce PCSK9 activity by inhibiting hepatic synthesis of PCSK9. Early results from ORION-1 of inclisiran, a chemically synthesized siRNA designed to target PCSK9 messenger RNA [58] given as double $300 \mathrm{mg}$ injection strategy, the first on day 1 and the second on day 90, reported a mean LDL-C reduction of $57 \%$ by day 120 and $52 \%$ reduction by day $180[59,60]$. New data reported that LDL-C reductions were sustained at 1 year with the two-dose $300 \mathrm{mg}$ regimen producing an average time adjusted fall of $46.8 \%$ across all dosing groups [61]. These results support a 6-month dosing interval which might improve adherence to therapy. These findings will help guide a phase III clinical outcome trial.

Another approach to reduce LDL-C is through the inhibition of cholesteryl ester transfer protein (CETP) but previous trials with CETP inhibitors have been disappointing. REVEAL (Randomized Evaluation of the Effects of Anacetrapib Through Lipid Modification) randomized 30,449 patients with prior CV disease already receiving intensive atorvastatin therapy with mean LDL cholesterol level of
$1.58 \mathrm{mmol} / \mathrm{l}$ to anacetrapib $100 \mathrm{mg}$ vs. placebo [62]. At a median follow-up of 4.1 years, anacetrapib was associated with a $9 \%$ reduction in the primary outcome of coronary death, MI, or coronary revascularization $(10.8 \%$ vs. $11.8 \%$; $P=0.004)$. Benefit from anacetrapib emerged after more than 3 years of treatment. Although CETP inhibitors produce large increases in HDL$\mathrm{C}$, the type of HDL-C produced is probably not atheroprotective and, rather, the observed $\mathrm{CV}$ benefit of anacetrapib in REVEAL appeared to correlate with the expected benefit derived from reduction in non-HDL cholesterol. Of note, unlike statins which may increase incidence of diabetes, anacetrapib was associated with a reduction new onset diabetes vs. placebo $(5.3 \%$ vs. $6.0 \%, P=0.05$ ) [62]. Ongoing follow-up will establish longer-term efficacy and safety. Drug cost, if approved, may help guide its use in the context of availability of efficacious but expensive PCSK9 pathway agents.

Epidemiological studies have suggested that higher levels of HDL-C may protect against CV disease. Prospective clinical trials of HDL-Craising agents have been disappointing to date, with some suggesting benefit on surrogate markers but with no clear reduction in $\mathrm{CV}$ event reduction attributable solely to HDL-C increase. CER-001 is a pre-beta HDL mimetic containing Apo A-1 and sphingomyelin. Early studies suggested potential for coronary plaque regression by infusing low dose CER-001 in high plaque burden acute coronary syndrome patients [63]. In the CER-001 Atherosclerosis Regression Acute Coronary Syndrome Trial (CARAT) 301 patients with acute coronary syndrome (ACS) and atheroma volume greater than 30\% determined via intravascular ultrasound (IVUS) were randomized to 10 weekly infusions of CER-001 at dose of $3 \mathrm{mg} / \mathrm{kg}$ or placebo [64]. IVUS was repeated 1-3 weeks after the last infusion. Disappointingly, there was no significant difference in the percentage atheroma volume between CER-001 (-0.09\%) and placebo $(-0.41 \%) P=0.15$, suggesting that this is not a useful strategy.

Current European guidelines recommend high intensity statin therapy for lipid lowering in patients with established coronary artery disease [65]. In Asia, the need for high vs. 
moderate intensity statin has been less certain. The REAL-CAD trial randomized 13,054 Japanese patients with stable coronary artery disease to high dose pitavastatin ( $4 \mathrm{mg}$ /day) or low dose pitavastatin ( $1 \mathrm{mg} /$ day). Although the study was stopped early because of dropouts, high intensity pitavastatin $4 \mathrm{mg} /$ day was associated with a $19 \%$ reduction in the primary endpoint of CV death, MI, stroke, or unstable angina (4.3\% vs. $5.4 \% ; P=0.01)[66]$, reduction in all cause death $(P=0.03)$, MI $(P=0.004)$, and any coronary revascularization $(P=0.008)$. Thus the benefits of maximally tolerated doses of statin appear to extend to a Japanese population.

It is increasingly recognized that chronic inflammatory conditions are associated with marked increases in CV risk and it has been postulated that some of the benefit of statins may be mediated through modest anti-inflammatory mechanisms [67]. CANTOS (Canakinumab Anti-inflammatory Thrombosis Outcome Study) provided the first definitive evidence that targeting vascular inflammation in the absence of reduction of LDL-C) is a further mechanism of reducing CV events [68]. CANTOS randomized 10,061 patients with a history of MI and inflammation as evidenced by elevated high sensitivity C-reactive protein (hsCRP) (median $4.20 \mathrm{mg} / \mathrm{l}$ ) to placebo or a subcutaneous injection every 3 months of canakinumab-a selective inhibitor of interleukin-1 beta (IL-1 $\beta$ ) and already approved in the USA for rare autoimmune diseases. Patients were otherwise well stabilized with $93.4 \%$ on high dose statins and two-thirds having undergone coronary intervention. The optimum canakinumab dose (150 mg) which lowered hs-CRP by $37 \%$ vs. placebo, but without any reduction in LDL-C, was associated with a $15 \%$ reduced risk for the composite primary endpoint of CV death, MI, or stroke $(P=0.0075$; Fig. 7$)$. Just as it appears "lower is better" for lipid lowering, patients in CANTOS who responded with an hs-CRP reduction greater than or equal to the median had a $27 \%$ reduction in the risk of major adverse cardiac events $(P=0.0001)$.

A further analysis from CANTOS presented at AHA 2017 found that participants allocated to canakinumab who achieved hsCRP $\leq 2 \mathrm{mg} / \mathrm{l}$ after a single dose had a $25 \%$ reduction in major

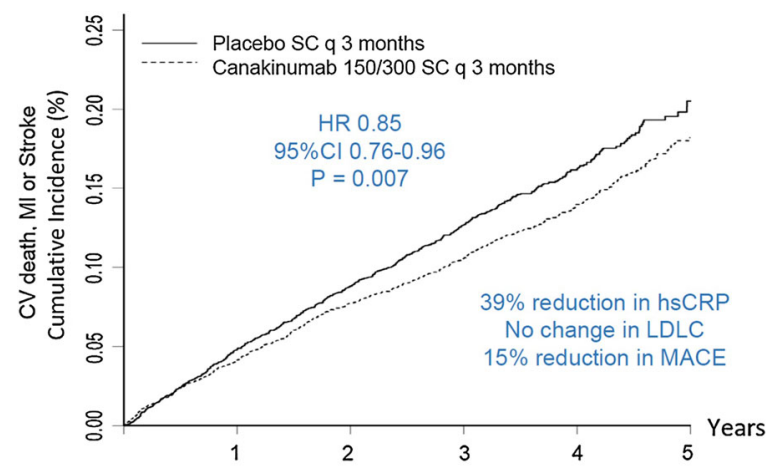

Fig. 7 Reduction of CV death, MI, or stroke with the IL$1 \beta$ inhibitor canakinumab vs. placebo in the CANTOS study

adverse $\mathrm{CV}$ events (multivariable adjusted $\mathrm{HR}$ $0.75, P<0.0001$ ) and $31 \%$ reduction in both CV mortality and all-cause mortality [69]. By contrast, effects were non-significant for all these endpoints amongst individuals with hsCRP $>2 \mathrm{mg} / \mathrm{l}$. This suggests that the magnitude of reduction and/or hsCRP achieved following a single dose may provide a useful method of identifying patients likely to derive benefit from continued treatment.

Canakinumab was associated with a small increase in deaths due to infection ( 0.31 vs. 0.18 events per 100 person-years; $P=0.02$ ), most commonly in older patients with diabetes. If canakinumab is approved for use in CV disease, patients will thus require monitoring for early signs of infection similar to that currently done for patients taking other biologic drugs.

The relationships between diet, CV disease, and mortality are controversial but represent areas of major public health interest. The Prospective Urban Rural Epidemiology (PURE) study is the largest prospective observational study to assess the association of dietary intake with CV disease and mortality with 135,335 individuals enrolled from 18 countries across five continents [70]. Participants were categorized into quintiles of nutrient intake. Higher carbohydrate intake was associated with higher risk of total mortality (highest quintile vs. lowest quintile HR 1.28, 95\% CI 1.12-1.46), but was not associated with higher risk of $\mathrm{CV}$ disease or $\mathrm{CV}$ mortality. Unexpectedly, lower risk of mortality was associated with higher intake of total 
fat and each type of fat (highest quintile vs. lowest quintile, total fat HR 0.77, 95\% CI 0.67-0.87). Saturated fat was associated with lower risk of stroke with total fat, saturated fat, and unsaturated fat found not to be significantly associated with risk of MI or CV mortality. These findings challenge current global dietary guidelines (based largely on western populations) and further research in this area is warranted.

\section{ADVANCES IN ACUTE CORONARY SYNDROMES AND ANTITHROMBOTIC THERAPY}

Supplemental oxygen is commonly given during acute MI, but latest ESC guidelines recommend reserving this for patients with oxygen saturations less than 90\% [71] with previous studies suggesting larger infarct size among STelevation MI patients who received oxygen [72]. The Determination of the Role of Oxygen in Suspected Acute Myocardial Infarction (DETO2X-AMI) trial enrolled 6629 patients in a registry-based randomized trial where patients with suspected MI and oxygen saturations $\geq 90 \%$ were assigned to receive oxygen at $6 \mathrm{l} / \mathrm{min}$ for $6-12 \mathrm{~h}$ vs. ambient air only [73]. No difference in all-cause mortality at 1 year was seen between the oxygen (5\%) and ambient air (5.1\%) groups, $P=0.80$; rates of rehospitalization with MI within 1 year were similar in both groups $(3.8 \%$ vs. $3.3 \%, P=0.33)$; and there was no difference in the extent of myocardial injury determined by cardiac troponin levels. This provides further support for avoiding supplemental oxygen therapy in the setting of acute MI in patients without hypoxemia.

ESC guidelines recommend 12-month dual antiplatelet therapy (DAPT) in STEMI patients undergoing primary PCI with DES [71]. For some patients a shorter duration of DAPT may be preferable so as to facilitate urgent surgery or in those with considerable bleeding risk. The DAPT-STEMI trial randomized patients with STEMI undergoing primary PCI with zotarolimus-eluting stent who were event-free at 6 months (no MI, stent thrombosis, target vessel or lesion revascularization, or stroke/bleeding requiring DAPT discontinuation) to either cessation of DAPT and continuation of aspirin only or a further 6 months of DAPT followed by single antiplatelet therapy with aspirin [74]. At 18-month follow up, incidence of the primary outcome of all-cause mortality, MI, revascularization, stroke, and TIMI major bleeding was similar between abbreviated 6-month DAPT and 12 -month DAPT courses $(4.8 \%$ vs. $6.6 \% P$ for non-inferiority $=0.004, \quad P$ for superiority $=0.26$ ). While the trial was underpowered to assess individual safety endpoints the results support consideration of shorter DAPT course in patients at high bleeding risk.

Prehospital administration of antiplatelet therapy is common practice in patients with STelevation MI and ESC guidelines advocate P2Y12 inhibitors at the time of first medical contact [71], although a previous study with ticagrelor did not show benefit with respect to TIMI flow, ST segment resolution, or MACE. A large retrospective observational study using data from the Swedish Coronary Angiography and Angioplasty Registry (SCAAR) evaluated patients undergoing primary PCI [75], the majority of whom were pretreated with P2Y12 inhibitors $(37,840$ pretreated; 6964 not pretreated). Pretreatment was not associated with any difference in mortality at 30 days $(5.2 \%$ vs. $7.6 \%$, adjusted odds ratio $1.07, P=0.31$ ), patency of infarct-related artery, or stent thrombosis at 30 days. Whilst lacking benefit, prehospital use of P2Y12 inhibitors appeared safe with no difference in risk of bleeding or neurological complications and in keeping with previous data [76].

Platelet function testing has not routinely been recommended to guide DAPT use as previous trials have failed to demonstrate improvement in clinical outcomes [77]. Nevertheless, de-escalation of antiplatelet therapy may be important in patients with elevated bleeding risk. The TROPICAL-ACS (Guided Deescalation of Antiplatelet Treatment in Patients with Acute Coronary Syndrome Undergoing Percutaneous Coronary Intervention) trial randomized 2610 ACS patients post PCI to a novel de-escalation protocol (1 week prasugrel, 1 week clopidogrel, and remaining on this unless high platelet reactivity guided switching back to 
prasugrel) vs. conventional prasugrel therapy [78]. The de-escalation strategy was non-inferior at 1 year for the primary endpoint of net clinical benefit [CV death, MI, stroke, or Bleeding Academic Research Consortium (BARC)] bleeding grade $\geq 2) 1$ year after randomization $(7.3 \%$ vs. $9.0 \%, P$ non-inferiority $=0.004)$. Whilst the sample size and number of events were modest, TROPICAL-ACS does suggest that de-escalation combined with platelet function testing to ensure sufficient platelet inhibition is logical, particularly in patients at highest bleeding risk.

One-year follow-up findings are now available from head-to-head PRAGUE 18 trial (Prasugrel vs. Ticagrelor in Patients with Acute Myocardial Infarction Treated with Primary Percutaneous Intervention) which randomized 1230 STEMI patients from 14 sites to prasugrel vs. ticagrelor. At 1 year, prasugrel and ticagrelor were found to have similar incidence for primary endpoint of $\mathrm{CV}$ death, stroke, re-infarction, or major bleeding $(6.6 \%$ vs. 5.7\%; $P=0.503$ ) [79]. Although the trial was likely underpowered, findings are in keeping with the current ESC guideline position supporting use of either drug in the STEMI setting.

Results from previous trials comparing bivalirudin with heparin in the acute coronary syndrome setting have been conflicting [80-82]. In Validate-Swedeheart (Bivalirudin vs. Heparin in ST-Segment and Non-ST-Segment Elevation MI in Patients on Modern Antiplatelet Therapy in the Swedish Web System for Enhancement and Development of Evidence-based Care in Heart Disease Evaluated According to Recommended Therapies Registry Trial) 6006 ACS patients (STEMI 3005, NSTEMI 3001) undergoing predominantly radial access PCI and receiving a potent P2Y12 inhibitor (either ticagrelor, prasugrel, or cangrelor) without planned use of glycoprotein IIb/IIIa inhibitors were randomized to bivalirudin vs. heparin during PCI [83]. There was no difference seen with bivalirudin for the primary endpoint of death, MI, or major bleeding at 180 days $(12.3 \%$ vs. $13.8 \%$, $P=0.54)$ and given the increased cost of bivalirudin this supports use of heparin for most ACS patients undergoing PCI in contemporary practice.
In patients with recent ACS taking DAPT, addition of low dose rivaroxaban $2.5 \mathrm{mg}$ bd has been shown to reduce mortality and ischemic events but to increase bleeding [84]. A modification of this approach was undertaken in GEMINI-ACS-1 (Safety of Rivaroxiban Versus Acetylsalicylic Acid in Addition to Either Clopidogrel or Ticagrelor Therapy in Participants with Acute Coronary Syndrome), in which patients with recent ACS taking P2Y12 inhibitor (clopidogrel or ticagrelor) were randomized to rivaroxaban $2.5 \mathrm{mg}$ bd vs. aspirin $100 \mathrm{mg}$ od [85]. The primary endpoint of TIMI non-CABG clinically significant bleeding was similar for rivaroxaban vs. aspirin $(5.3 \%$ vs. $4.9 \%, P=0.58)$ and ischemic endpoints were similar (although the trial was not powered to assess these fully). Further evaluation of efficacy and safety of this dual antithrombotic regimen in a larger phase III trial would appear worthwhile.

Patients with stable CV disease remain at risk of recurrent events despite use of secondary prevention strategies. Extending DAPT beyond 1 year post ACS with aspirin and reduced-dose ticagrelor (60 $\mathrm{mg}$ bd) has been shown to reduce the composite of CV death, MI, or stroke but increases major bleeding and is best used in patients at lower bleeding risk [86]. Anticoagulation monotherapy has not shown net clinical benefit over aspirin alone, and a previous trial of aspirin plus full-dose apixaban [87] was halted because of excessive bleeding. COMPASS (Cardiovascular Outcomes for People Using Anticoagulation Strategies) randomized 27,395 patients with stable atherosclerotic vascular disease (coronary or peripheral arterial disease) to one of three groups: rivaroxaban $2.5 \mathrm{mg}$ bd plus aspirin $100 \mathrm{mg}$ od; rivaroxaban $5 \mathrm{mg}$ bd alone; or aspirin $100 \mathrm{mg}$ od alone. The trial was stopped early at 23 months at which point CV death, MI, or stroke was reduced by $24 \%$ in those receiving the combination treatment vs. aspirin alone ( 4.1 vs. $5.4 \% ; P<0.001$; Fig. 8 ).

Bleeding was increased with the combination strategy $(3.1 \%$ vs. $1.9 \% ; P<0.001)$ but there was no significant increase in fatal or intracranial bleeding. Combination treatment was associated with reduction in net clinical events (death, MI, stroke, or fatal/critical bleed 


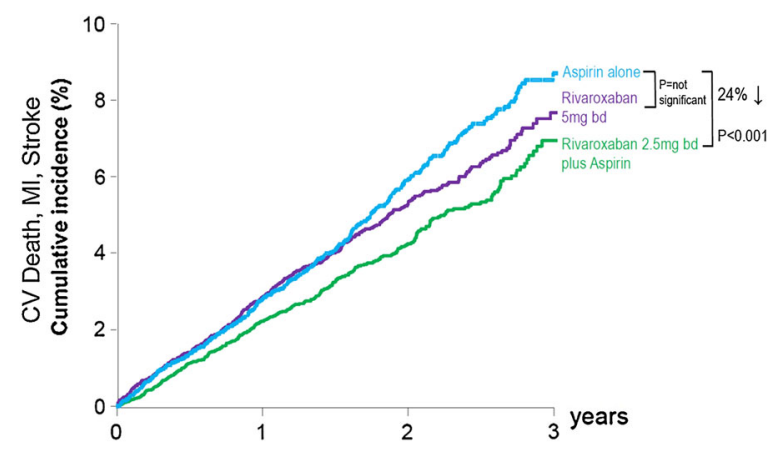

Fig. 8 Reduction of CV death, MI, or stroke with the combination of very low dose rivaroxaban plus aspirin vs. aspirin alone in the COMPASS study

$4.7 \%$ vs. $5.9 \% ; P<0.001)$, CV death $(1.7 \%$ vs. $2.2 \% ; P=0.02)$, and death from any cause (3.4\% vs. $4.1 \% ; P=0.01$ ) [88]. In contrast, use of rivaroxaban $5 \mathrm{mg}$ bd alone vs. aspirin alone was not associated with a reduction in the primary outcome but did increase bleeding compared with aspirin alone, with no significant improvement in net clinical benefit. The results of COMPASS are likely to change practice guidelines. Defining the appropriate patient for combination therapy will require careful consideration of the balance of ischemic and bleeding risks especially in older patients and people with renal disease, where bleeding risk is a specific concern. Further data analysis will likely inform treatment guidelines, but those at highest baseline absolute risk such as those with concurrent peripheral arterial disease are likely to have most to gain [89].

The optimum management of patients post PCI who also have concurrent AF requiring anticoagulation is an area of current interest. Previously, PIONEER-AF reported lower rates of clinically significant bleeding but no excess in ischemic events with reduced-dose rivaroxaban $15 \mathrm{mg}$ od plus a single P2Y12 inhibitor for 12 months or with very low dose rivaroxaban $2.5 \mathrm{mg}$ bd plus DAPT for 1,6 , or 12 months vs. standard triple therapy (warfarin plus DAPT for 1,6 , or 12 months) [90]. New data have now been reported from REDUAL-PCI (Randomized Evaluation of Dual Antithrombotic Therapy with Dabigatran vs. Triple Therapy with Warfarin in Patients with Nonvalvular Atrial Fibrillation Undergoing Percutaneous Coronary
Intervention) which randomized 2725 patients with non-valvular AF undergoing PCI to dual therapy with dabigatran $110 \mathrm{mg}$ bd plus P2Y12 inhibitor vs. dual therapy with dabigatran $150 \mathrm{mg}$ bd plus P2Y12 inhibitor vs. standard triple therapy group (warfarin plus DAPT) [91]. Dabigatran $110 \mathrm{mg}$ bd plus P2Y12 inhibitor was associated with a $43 \%$ reduction in major or clinically relevant non-major bleeding events vs. triple therapy $(15.4 \%$ vs. $26.9 \%$; $P$ superiority $<0.001$; Fig. 9). Bleeding with dabigatran $150 \mathrm{mg}$ bd plus P2Y12 inhibitor was numerically lower and non-inferior but not superior to triple therapy (20.2\% vs. $25.7 \%)$. There was no significant increase in ischemic events, although the study was not sufficiently powered to test this. The benefit of a dual therapy approach was consistent across subgroups including ACS, use of DES, and use of more potent P2Y12 (although ticagrelor was only used in 12\%) [92]. Notably, both dabigatran doses were full anticoagulation doses approved for use in stroke prevention and DC cardioversion, whereas Pioneer-AF used reduced-dose rivaroxaban. While further studies are ongoing with apixaban and edoxaban, RE-DUAL suggests that dual therapy rather than conventional triple therapy may be preferable for most patients with AF undergoing PCI.

The optimal strategy for maintenance of saphenous vein graft (SVG) patency in patients undergoing CABG is hotly debated. The DACAB trial (Compare the Efficacy of Different Antiplatelet Therapy Strategy After Coronary Artery Bypass Graft Surgery) [93] randomized 500 patients to aspirin $100 \mathrm{mg}$ monotherapy vs. ticagrelor $90 \mathrm{mg}$ bd monotherapy vs. DAPT with aspirin plus ticagrelor. DAPT was associated with highest SVG patency at 1 year (Table 2; $P=0.0006$ vs. aspirin monotherapy) but this benefit was offset by significantly increased bleeding. In addition, $2 / 3$ of patients undergoing CABG had ACS in whom benefits of DAPT might be greater, and the benefit in an elective stable population is uncertain.

The original 2014 POISE 2 (Perioperative Ischemic Evaluation 2) trial reported that perioperative aspirin during undergoing non-cardiac surgery did not reduce death or MI but did increase major bleeding. However, aspirin may 

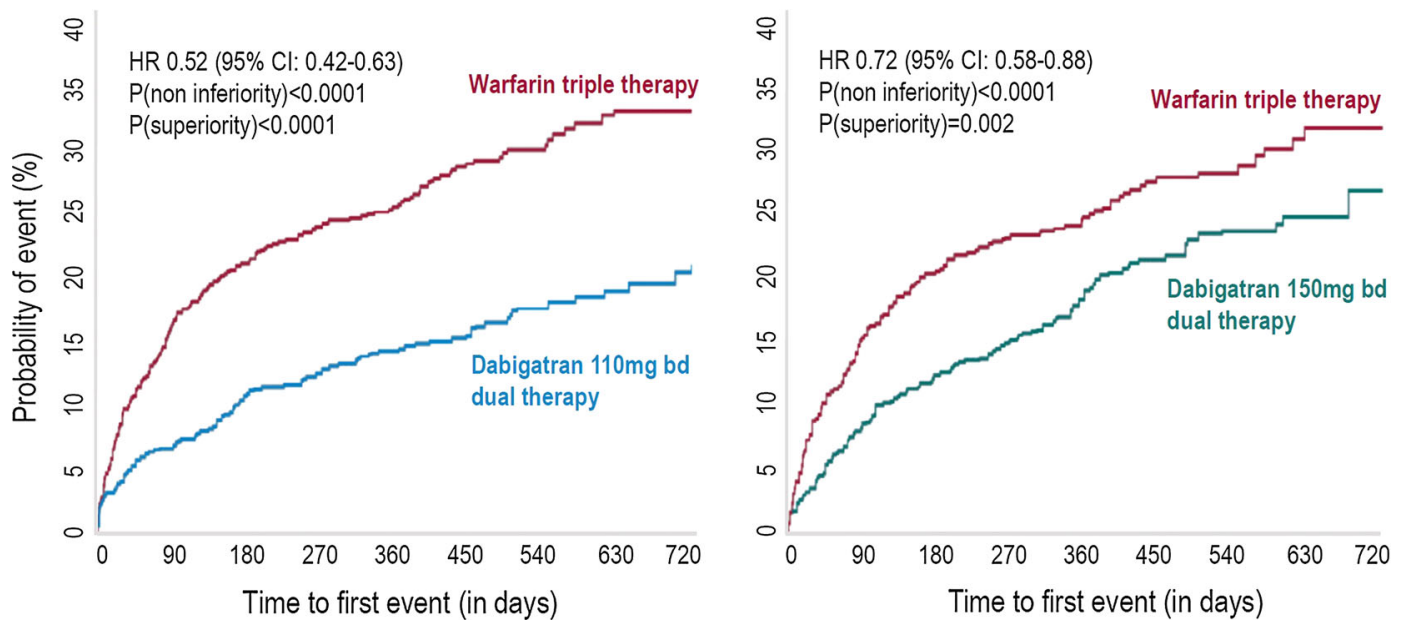

Fig. 9 Reduction in clinically relevant non-major bleeding with dabigatran compared to warfarin in RE-DUAL PCI

Table 2 Summarized results of DACAB trial

\begin{tabular}{llll}
\hline Outcome & $\begin{array}{l}\text { Aspirin } \\
\mathbf{1 0 0} \mathbf{~ m g} \\
(\%)\end{array}$ & $\begin{array}{l}\text { Ticagrelor } \\
\mathbf{9 0} \mathbf{~ m g} \text { bd } \\
(\%)\end{array}$ & $\begin{array}{l}\text { Aspirin + ticagrelor } \\
(\%)\end{array}$ \\
\hline $\begin{array}{c}\text { SVG } \\
\text { patency }\end{array}$ & 76.5 & 82.8 & 88.7 \\
$\begin{array}{c}\text { SVG non- } \\
\text { occlusion }\end{array}$ & 80.6 & 86.1 & 89.9 \\
$\begin{array}{c}\text { Non- } \\
\text { CABG }\end{array}$ & 9 & 12.1 & 30.4 \\
bleeding & & & \\
$\begin{array}{c}\text { Major } \\
\text { bleeding }\end{array}$ & 0 & 0.6 & 1.2 \\
$\begin{array}{c}\text { Non- } \\
\text { CABG }\end{array}$ & 0 & 1.2 & 1.8 \\
$\begin{array}{l}\text { and } \\
\text { CABG } \\
\text { bleeding }\end{array}$ & & & \\
\hline
\end{tabular}

be of greater importance in those with previous PCI. Within the POISE- 2 cohort, 470 patients were identified with previous PCI (but $>1$ month after bare metal stent and $>1$ year after DES) [94]. In previous PCI patients, aspirin vs. placebo was associated with a marked reduction in MI (5.1\% vs. $11.0 \%$; HR $0.44 ; 95 \%$
CI 0.22-0.87), whereas there was no benefit in those without PCI $(P$ interaction $=0.021)$. PCI vs. non-PCI subgroups did not show a significant $P$ interaction value regarding bleeding (and thus the small increase in bleeding noted in the overall trial might still be seen in the PCI subgroup). Although the PCI subgroup was small compared with the overall trial, these findings support current practice of continuing aspirin for patients with previous PCI undergoing noncardiac surgery.

\section{ADVANCES IN ATRIAL FIBRILLATION AND ELECTROPHYSIOLOGY}

Despite increased utilization of non-vitamin K oral anticoagulants (NOACs) in venous thromboembolism (VTE) and AF settings, there is still a need for large real-world datasets to assess safety outside the controlled environment of randomized trials. Hemmelgarn et al. undertook a large observational study comparing 12,489 patients receiving NOACs (mostly rivaroxaban) vs. 47,036 receiving warfarin for VTE [95]. No significant difference was reported in major bleeding or in death between NOAC vs. warfarin, although follow-up was relatively short (mean 85 days).

Longer-term safety of NOAC for VTE was assessed in EINSTEIN-CHOICE (Rivaroxaban or Aspirin for Extended Treatment of Venous 
Thromboembolism) [96] which randomized 3396 patients with proven VTE following an initial 6- to 12-month course of full anticoagulation to a further 12 months of rivaroxaban $20 \mathrm{mg}$ vs. rivaroxaban $10 \mathrm{mg}$ vs. aspirin $100 \mathrm{mg}$. Rivaroxaban was associated with a significant reduction in the primary efficacy outcome of symptomatic recurrent fatal or nonfatal VTE but no significant difference in major bleeding or in clinically relevant minor bleeding (Fig. 10). Whilst further studies are required to determine safety and optimal dosing, this trial adds further support to the previously published AMPLIFYEXT (Apixaban for Extended Treatment of Venous Thromboembolism) trial and reassures clinicians about the safety and efficacy of extended duration NOACs [97].

Previous trials for management of recurrent reflex vasovagal syncope have been largely disappointing. The role of cardiac pacing remains uncertain except when bradycardia is the documented predominant feature (reflected in the current AHA syncope guidelines with a IIb indication for pacing) [98]. Closed loop stimulation (CLS) pacing was the focus of the small prospective SPAIN (Closed Loop Stimulation for Neuromediated Syncope) trial [99] in 46 patients with high burden syncope ( $\geq 5$ episodes, $\geq 2$ episodes in the past year), and a cardioinhibitory head-up tilt test (bradycardia $<40$ beats/min for $10 \mathrm{~s}$ or asystole $>3 \mathrm{~s}$ ). Patients were randomized to 12 months DDD mode-CLS pacing then 12 months sham DDI mode pacing vs. 12 months sham DDI mode then 12 months DDD mode-CLS pacing. DDD
mode-CLS pacing was associated with a significant reduction in syncope burden and a sevenfold reduction in recurrence (8.7\% vs. $45.7 \%)$. These results are of potential importance and if replicated in the BioSync CLS trial will likely lead to revision of syncope guidelines.

Early identification of $\mathrm{AF}$ is of high clinical importance. REHEARSE AF (Assessment of Remote Heart Rhythm Sampling Using the AliveCor Heart Monitor to Screen for Atrial Fibrillation) [100] randomized 1001 patients without known AF, mean age 73 years, and CHADSVASC $\geq 2$ to use of iECG (AliveCor device attached to iPod to transmit $30 \mathrm{~s}$ ambulatory ECGs twice weekly or when symptomatic) vs. routine care (physician review) for 12 months. Use of the ambulatory iECG was associated with an almost fourfold increase in detection of AF (19 vs. $5 ; P=0.0007)$. The incidence of stroke, TIA, or systemic embolism did not reach significance (6 vs. $10 ; P=0.34$ ), although the study was not powered for these outcomes. The cost per AF diagnosis in REHEARSE was relatively high $(£ 8255 / \$ 10,780)$ but reduction in cost may be possible by using patients' own smartphones and further algorithm improvement to reduce need for clinician over-reading.

Patient adherence to anticoagulation treatment for AF can be poor. IMPACT AF (A Multifaceted Intervention to Improve Treatment with Oral Anticoagulants in Atrial Fibrillation) cluster randomized 2281 patients from five countries (Argentina, Brazil, China, India, and Romania) to a quality improvement

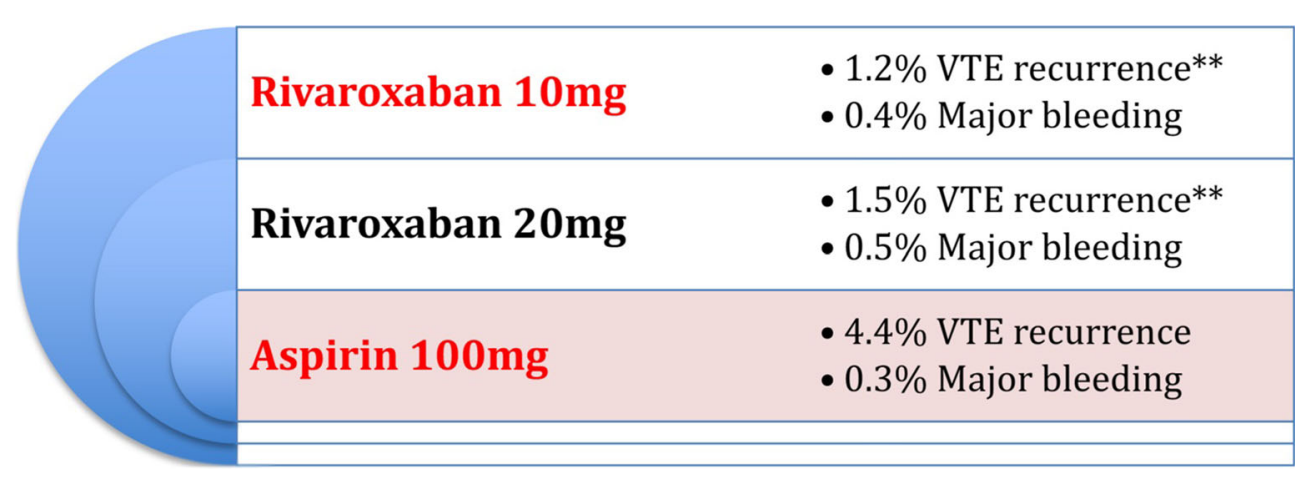

Fig. 10 Percentage VTE recurrence/major bleeding in three trial arms, rivaroxaban $10 \mathrm{mg}$, rivaroxaban $20 \mathrm{mg}$, aspirin $100 \mathrm{mg} .{ }^{* *} P<0.001$ vs. aspirin 
educational interventional for patients and clinicians vs. usual care. Educational intervention vs. usual care was associated with an absolute 9.1\% (95\% CI 3.8-14.4) improvement in adherence and a reduction in stroke (HR 0.48, 95\% CI 0.23-0.99; log-rank $P$ value $=0.0434)[101]$.

Conventional anticoagulation protocols advise at least 3 weeks pretreatment unless the $\mathrm{AF}$ is of short duration or imaging excludes left atrial thrombus. The efficacy of shorter duration anticoagulation prior to DC cardioversion is unclear. A short duration of anticoagulation $(<48 \mathrm{~h})$ prior to DC cardioversion (continuing for a month post cardioversion) was tested in EMANATE (Eliquis evaluated in acute cardioversion coMpared to usuAl treatmeNts for AnTicoagulation in subjEcts with NVAF) [102] which randomized 1500 anticoagulation-naïve patients (mean age 65 years, mean CHADSVASC 2.4) to apixaban vs. IV heparin transitioning to warfarin. In the apixaban group, cardioversion less than $2 \mathrm{~h}$ after additional pre-loading with double usual dose apixaban was permitted at clinician discretion. At follow-up, no stroke or systemic embolism events occurred in the apixaban group vs. 6 patients $(0.8 \%)$ of the heparin/warfarin group $(P=0.016)$. Bleeding rates were similar. Of note, around half of patients underwent pre-procedure imaging at clinician discretion showing left atrial appendage thrombus in $7 \%$ which persisted, despite a further 1 month of anticoagulation, in over half of patients. In summary, EMANATE suggests that apixaban is a reasonable alternative to warfarin in DC cardioversion (it was not powered for superiority), and provides further data regarding short duration pretreatment to that reported with rivaroxaban and edoxaban $[103,104]$. However, since $7 \%$ of patients undergoing pre-cardioversion imaging had evidence of thrombus, it does continue to support pre-cardioversion imaging where feasible if a short duration anticoagulation is being considered.

$\mathrm{AF}$ in heart failure patients is associated with increased mortality and morbidity but maintenance of sinus rhythm following cardioversion can be difficult. RACE 3 (Routine vs. Aggressive Upstream Rhythm Control for Prevention of
Early Atrial Fibrillation in Heart Failure) [105] randomized 250 patients with mild to moderate impairment in left ventricular ejection fraction (LVEF) and early persistent AF scheduled for DC cardioversion to upstream treatment (cardiac rehabilitation, mineralocorticoid receptor antagonist, statin, ACEI/ARB) vs. usual care for at least 3 weeks prior to DC cardioversion. At 12 months, upstream treatment was associated with a $19 \%$ improvement in maintenance of sinus rhythm $(75 \%$ vs. $63 \%, P=0.021)$ as assessed by 7-day Holter. RACE 3 thus supports upstream risk factor modification at least for heart failure patients with $\mathrm{AF}$ as part of a rhythm control strategy.

Catheter ablation vs. drug therapy in appropriate patients is recognized to reduce AF burden endpoints, but since improvement in symptoms is the primary indication for rhythm control, quality of life may be a more appropriate endpoint. CAPTAF (Catheter Ablation compared with optimized Pharmacological Therapy for Atrial Fibrillation) [106] randomized 155 patients with symptomatic AF who had failed one drug to ablation with pulmonary vein isolation vs. antiarrhythmic drug therapy. At 12 months, ablation vs. drug therapy was associated with a greater improvement in quality of life score as assessed by the short form 36 item health survey (11.0 vs. 3.1 change in score; $P=0.0084)$. AF burden assessed by implantable cardiac monitor was numerically reduced in the ablation group this did not reach statistical significance.

CASTLE AF (Catheter ablation vs. Standard conventional Treatment in patients with Left ventricular systolic dysfunction an Atrial Fibrillation) is the first trial to assess the hard outcome of mortality or heart failure hospitalization in patients with reduced LVEF [107]. CASTLE AF randomized 397 patients with persistent or paroxysmal $\mathrm{AF}, \mathrm{LVEF} \leq 35 \%$, NYHA class $\geq 2$, ICD or CRTD implant, and failed at least one anti-arrhythmic to catheter ablation by pulmonary vein isolation (Fig. 11) vs. conventional therapy. Ablation was associated with a $38 \%$ reduction in the composite of death/heart failure hospitalization $(28.5 \%$ vs. $44.6 \%$, HR $0.62, P=0.007), 47 \%$ reduction in all-cause mortality (HR 0.52; 95\% CI 0.32-0.86; 


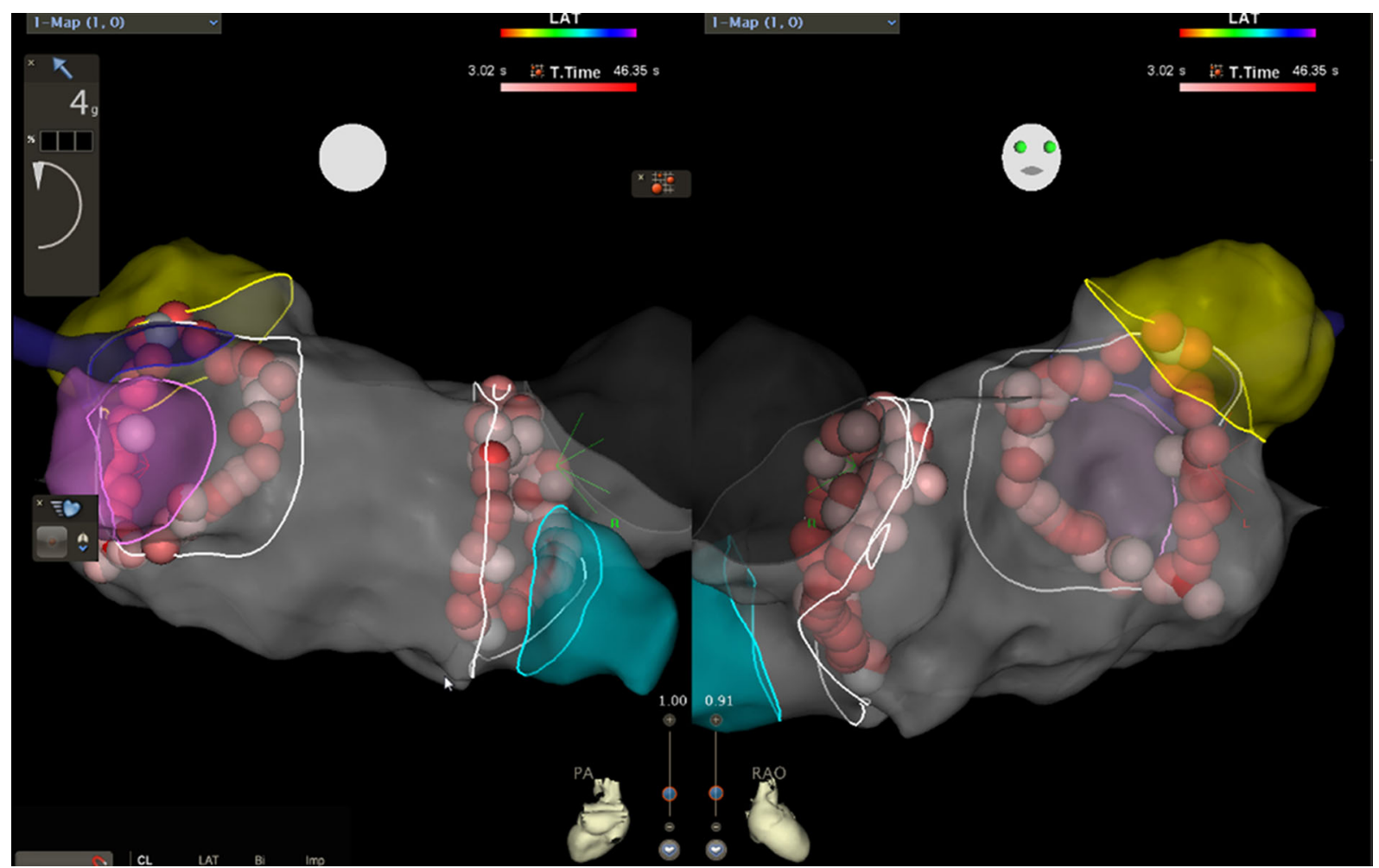

Fig. 11 Isolated pulmonary veins using cryotherapy with aid of CARTO mapping system

$P=0.011)$, and $51 \%$ reduction in CV mortality (HR $0.49,95 \%$ CI $0.29-0.84, P=0.008$ ). Also of interest, by 5 years, LVEF had improved by $8 \%$ in the ablation group vs. $0 \%$ in the conventional group. Similar LVEF findings were also reported in CAMERA-MRI (Catheter Ablation vs. Medical Rate Control in Atrial Fibrillation and Systolic Dysfunction) trial which randomized 301 patients with idiopathic cardiomyopathy and $\mathrm{EF} \leq 45 \%$ to catheter ablation or rate control. At 6 months, ablation was associated with significantly greater absolute improvement in LVEF assessed by MRI $(18 \pm 13 \%$ vs. $4 \pm 13 \% ; P<0.0001)$ [108]. It remains uncertain whether the results obtained from these small trials of highly selected patients can be extrapolated to the general population or those with differing etiologies of LVEF impairment. However, they do lend support to the increasing evidence of ablation being a safe and beneficial strategy for patients with $\mathrm{AF}$ and reduced LVEF.

Pocket hematoma following device implantation has previously been shown to be reduced by $81 \%$ using uninterrupted warfarin vs. interruption with heparin bridging BRUISE-CONTROL 1 (A Randomized Controlled Trial of Continued vs. Interrupted Direct Oral AntiCoagulant at the Time of Device Surgery) [109]. As NOACs are increasingly preferred anticoagulant treatment, BRUISE-CONTROL 2 [110] randomized 662 patients to uninterrupted NOAC vs. interrupted NOAC (stopping $\geq 2$ days pre-procedure and resuming $\geq 24 \mathrm{~h}$ post procedure). In this study, uninterrupted NOAC did not show any difference in incidence of pocket hematoma vs. interrupted NOAC (both 2.1\%). Unfortunately, the study was stopped early because of futility based on the low event rate (trial powered for 16\%), although this lower rate might have been predicted since, unlike BRUISE-CONTROL 1, heparin bridging was not used in the interrupted NOAC arm. Nevertheless, BRUISE-CONTROL 2 suggests it is reasonable to undertake device implantation immediately rather than waiting for 2 days of NOAC interruption in urgent clinical scenarios such as complete heart block. 
Prior to AF ablation, uninterrupted dabigatran has been shown to have comparable stroke risk reduction but lower bleeding risk than uninterrupted warfarin. Since the bleeding events seen with dabigatran mainly occurred in those with final dabigatran dose less than $8 \mathrm{~h}$ pre-procedure, use of "minimally" interrupted dabigatran was tested in ABRIDGE-J (ABlation perioperative DabiGatran in use Envisioning in Japan) [111] which randomized 500 patients to minimally interrupted dabigatran (omitting 1-2 doses; with heparin bridging if $>24 \mathrm{~h}$ ) vs. uninterrupted warfarin. Minimally interrupted dabigatran was associated with fewer major bleeds $(1.4 \%$ vs. $5.0 \% P=0.032)$ but no excess of cerebrovascular events. These data might support a modification of the current practice of continued anticoagulation during AF ablation, although the study was underpowered to detect stroke events; thus, further trials would be helpful.

\section{ADVANCES IN HEART FAILURE}

Low cardiac output syndrome occurs in $3-14 \%$ of patients undergoing cardiac surgery on cardiopulmonary bypass [112]. Levosimendan increases cardiac contractility by calcium sensitization of troponin $\mathrm{C}$ (in addition to vasodilation and cardioprotection related to the opening of sarcolemmal and mitochondrial potassium-ATP channels, respectively) and has previously been reported to improve cardiac index [113]. The largest levosimendan trial to date, LEVO CTS (Levosimendan in Patients with Left Ventricular Dysfunction Undergoing Cardiac Surgery) [112], randomized 849 patients with LVEF $<35 \%$ undergoing cardiopulmonary bypass to pre and post levosimendan vs. placebo. Although levosimendan was associated with a significant improvement in cardiac index (2.86 vs. $2.68, P<0.001$ ), and lower incidence of low cardiac output syndrome $(18.2 \%$ vs. $25.7 \% ; P=0.007)$, this did not translate to improvement in the primary composite endpoint of 30-day death, renal replacement therapy, perioperative MI, or mechanical device support. There was a trend to reduced death at 90 days $(4.7 \%$ vs. $7.1 \% ; P=0.12)$. Although levosimendan was relatively well tolerated, its role during cardiac surgery remains unclear and further studies in a less heterogeneous population may be beneficial.

The ESC hypertrophic cardiomyopathy (HCM) risk-sudden cardiac death (SCD) calculator was derived from a relatively restricted population and thus its applicability to other populations has been uncertain [114]. The HCM-EVIDENCE study [115] retrospectively assessed the predictive value of the ESC HCMSCD calculator among 3703 patients from a larger, geographically diverse cohort population. The predicted risk of SCD at 5 years (based on the HCM-SCD calculator) and the observed risk tracked well in high risk and low risk subgroups (high risk predicted $\geq 6 \%$; observed $8.9 \%$; low risk predicted $<4 \%$, observed $1.4 \%$ ). The ESC HCM-SCD calculator was thus confirmed as a suitable tool to guide implantation or deferral of ICD in high and low risk groups, respectively. Decision-making in intermediate risk patients $(4 \%$ to $<6 \%)$ remains less clearHCM-EVIDENCE observed similar 5-year outcomes to low risk patients, suggesting that conservative management is reasonable for most (but ICDs may prevent SCD in some, especially those at $\geq 5 \%$ predicted risk) [115].

Early use of a mineralocorticoid receptor antagonist (MRA) 1-3 days post MI has previously been shown to reduce mortality in patients with reduced LVEF [116] but not in those with preserved LVEF [117, 118]. Beygui et al. presented a new pooled analysis of data from two previous preserved LVEF studies-the large STEMI subset of ALBATROSS (Early Aldosterone Blockade in Acute Myocardial Infarction) which compared spironolactone $25 \mathrm{mg}$ vs. placebo (STEMI subset of [117]) and REMINDER (Impact of Eplerenone on Cardiovascular Outcomes in Patients Post Myocardial Infarction) which compared eplerenone $25-50 \mathrm{mg}$ vs. placebo in STEMI [118]. At 190-day follow-up, use of MRA was associated with a marked reduction in mortality $(0.4 \%$ vs. $1.6 \% ; P=0.006)$. The analysis can only be considered exploratory in nature, and does not address use of MRA following NSTEMI, but does support further prospective study in this area 
such as the planned large CLEAR-SYNERGY study.

Increasingly patients with advanced heart failure are receiving left ventricular assist devices (LVAD) as destination therapy. The decision to implant is often difficult and associated with significant psychological stress for the patient. DECIDE LVAD trial (Trial of a Decision Support Intervention for Patients and Caregivers Offered Destination Therapy Heart Assist Device) randomized 248 patients to structured decisionmaking intervention with clinical education, pamphlet, and video decision aids vs. standard care [119]. Decision support intervention was associated with reduction in implantation rates (53.9\% vs. $79.9 \% ; P=0.008)$, improved decision quality but with no difference in decision conflict, regret, or quality of life. This trial highlights the importance of appropriate patient education to enable informed decisionmaking.

Treatment of heart failure with preserved ejection fraction (HFpEF) remains a challenge with discouraging results from previous pharmacological trial interventions. The role of reduction in left atrial pressure and pulmonary capillary wedge pressure (PCWP) was assessed in REDUCE LAP HF (A Transcatheter Interatrial Shunt Device for the Treatment of Heart Failure with Preserved Ejection Fraction) randomized 44 patients with NYHA class III-IV, LVEF > $40 \%$, exercise PCWP $\geq 25 \mathrm{mmHg}$, and PCWP to right atrial pressure gradient $\geq 5 \mathrm{mmHg}$ to an 8-mm-diameter interatrial shunt inserted via transfemoral route vs. a double-blind sham procedure [120]. At 1 month, intervention was associated with a $3.2-\mathrm{mmHg}$ reduction in exercise PCWP at $20 \mathrm{~W}$ (vs. 0.9 gain with sham; $P=0.028$ ), with no major CV or cerebrovascular adverse events. The concept of this phase II trial, whereby pressure is transferred to the right heart where it can be more easily treated by diuretics, is encouraging and larger trials are being considered to assess impact on symptoms and prognosis.

Latest data evaluating stem cells for the elusive goal of myocardial tissue regeneration were presented at AHA 2017. The ALLSTAR (Allogenic Heart Stem Cells to Achieve Myocardial Regeneration) trial [121] randomized 142 patients with $\mathrm{MI}<12$ months and $\mathrm{EF}<45 \%$ in 2:1 fashion to intracoronary allogeneic cardiosphere-derived stem cell injection vs. placebo. Disappointingly, stem cell injection was not associated with any reduction in infarct size or LV volume as assessed by MRI. However, the HOPE-Duchenne trial (Halt Cardiomyopathy Progression in Duchenne) [122], which randomized 25 male patients with Duchenne muscular dystrophy ( $2 / 3$ wheelchair bound) to the same intracoronary allogenic stem cell treatment vs. placebo, reported a 7\% reduction in myocardial scar on MRI at 1 year $(P=0.03)$, whereas placebo showed increased scar as is typical in Duchenne (between-group difference $11.9 \%$ at 1 year). Skeletal muscle function scores improved in those with more advanced disease. A small troponin rise was noted in all patients receiving intracoronary treatment and transient AF in 5/13 (although AF is commonly seen in Duchenne patients undergoing cardiac catheterization). Although a small trial, given this positive signal, the HOPE- 2 phase 2 trial is now planned, which will assess patients receiving cardiosphere-derived stem cell via intravenous infusion.

\section{CONCLUSION}

In this paper we have highlighted and summarized numerous important studies in the field of cardiology which have been published and/or presented to major international cardiology meetings throughout 2017. Many of these studies will contribute to updating of current practice guidelines and others will play an integral role in the advancement and development of new therapeutic strategies.

\section{ACKNOWLEDGMENTS}

Funding. No funding or sponsorship was received for this study or publication of this article.

Authorship. All named authors meet the International Committee of Medical Journal 
Editors (ICMJE) criteria for authorship for this manuscript, take responsibility for the integrity of the work as a whole, and have given final approval for the version to be published.

Disclosures. Conor McQuillan, Alistair Gray, and Aileen Kearney have nothing to disclose. Ian B. A. Menown has received grants to institution, honoraria and/or conference sponsorship from Biosensors, Boston Scientific, Meril Life, Orbus Neich, Astra Zeneca, Amgen, Bayer, Boehringer Ingelheim, Daichii Sankyo, Lilly, Bristol Myers Squibb, Pfizer, and Sanofi Aventis.

Compliance with Ethics Guidelines. This article is based on previously conducted studies and does not involve any new studies of human or animal subjects performed by any of the authors.

Open Access. This article is distributed under the terms of the Creative Commons Attribution-NonCommercial 4.0 International License (http://creativecommons.org/licenses/ by-nc/4.0/), which permits any noncommercial use, distribution, and reproduction in any medium, provided you give appropriate credit to the original author(s) and the source, provide a link to the Creative Commons license, and indicate if changes were made.

\section{REFERENCES}

1. Smits PC, Abdel-Wahab M, Neumann FJ, et al. Fractional flow reserve-guided multivessel angioplasty in myocardial infarction. $\mathrm{N}$ Eng $\mathrm{J}$ Med. 2017;376(13):1234-44.

2. Gotberg M, Christiansen EH, Gudmundsdottir IJ, et al. Instantaneous wave-free ratio versus fractional flow reserve to guide PCI. $\mathrm{N}$ Eng $\mathrm{J}$ Med. 2017;376(19):1813-23.

3. Davies JE, Sen S, Dehbi HM, et al. Use of the instantaneous wave-free ratio or fractional flow reserve in PCI. N Eng J Med. 2017;376(19):1824-34.

4. Brilakis E. CrossBoss first: a randomized trial of antegrade dissection and re-entry vs standard wire escalation for crossing coronary artery chronic total occlusions. Presented at transcatheter therapeutics conference; October 31, 2017; Denver, Colorado.
5. Park SJ. Drug-eluting stent versus optimal medical therapy in patients with coronary chronic total occlusion: DECISION CTO randomized trial. Presented at American College of Cardiology conference; March 18, 2017; Washington DC.

6. Mashayekhi K. REVASC: a randomized trial to assess recovery of left ventricular function after PCI of coronary artery chronic total occlusions. Presented at transcatheter therapeutics conference; October 31, 2017; Denver, Colorado.

7. Ibanez B, James S, Agewall S, et al. 2017 ESC guidelines for the management of acute myocardial infarction in patients presenting with ST-segment elevation: the task force for the management of acute myocardial infarction in patients presenting with ST-segment elevation of the European Society of Cardiology (ESC). Eur Heart J. 2017;39(2):119-77.

8. Thiele H, Akin I, Sandri M, et al. PCI strategies in patients with acute myocardial infarction and cardiogenic shock. N Eng J Med. 2017;377(25):2419-32.

9. Gray A, McQuillan C, Menown IB. Advances in clinical cardiology 2016: a summary of the key clinical trials. Adv Ther. 2017;34(7):1503-27.

10. Baron SJ, Chinnakondepalli K, Magnuson EA, et al. Quality of life after everolimus-eluting stents or bypass surgery for treatment of left main disease. J Am Coll Cardiol. 2017;70(25):3113-22.

11. Chen SL, Zhang JJ, Han Y, et al. Double kissing crush versus provisional stenting for left main distal bifurcation lesions: DKCRUSH-V randomized trial. J Am Coll Cardiol. 2017;70(21):2605-17.

12. Al-Lamee R, Thompson D, Dehbi HM, et al. Percutaneous coronary intervention in stable angina (ORBITA): a double-blind, randomised controlled trial. Lancet. 2017;391(10115):31-40.

13. Fearon WF, Nishi T, De Bruyne B, et al. Clinical outcomes and cost-effectiveness of fractional flow reserve-guided percutaneous coronary intervention in patients with stable coronary artery disease: three-year follow-up of the FAME 2 trial (fractional flow reserve versus angiography for multivessel evaluation). Circulation. 2018;137(5):480-7.

14. Kandzari DE, Mauri L, Koolen JJ, et al. Ultrathin, bioresorbable polymer sirolimus-eluting stents versus thin, durable polymer everolimus-eluting stents in patients undergoing coronary revascularisation $(\mathrm{BIOFLOW} \mathrm{V})$ : a randomised trial. Lancet. 2017;390(10105):1843-52.

15. Kereiakes DJ, Ellis SG, Metzger C, et al. 3-year clinical outcomes with everolimus-eluting bioresorbable coronary scaffolds: the ABSORB III trial. J Am Coll Cardiol. 2017;70(23):2852-62. 
16. Baan J Jr, Claessen BE, Boerlage-van Dijk K, et al. A randomized comparison of paclitaxel-eluting balloon versus everolimus-eluting stent for the treatment of any in-stent restenosis: the DARE trial. JACC Cardiovasc Interv. 2017;11(3):275-83.

17. Suryapranata H. REDUCE: a randomized trial of 3-month vs 12-month DAPT after implantation of a bioabsorbable polymer-based metallic DES with a luminal CD34+ antibody coating in patients with ACS. Presented at transcatheter therapeutics conference; November 1, 2017; Denver, Colorado.

18. Vlastra W, Delewi R, Sjauw KD, et al. Efficacy of the RADPAD protection drape in reducing operators' radiation exposure in the catheterization laboratory: a sham-controlled randomized trial. Circ Cardiovasc Interv. 2017. https://doi.org/10.1161/ CIRCINTERVENTIONS.117.006058.

19. Mazer CD, Whitlock RP, Fergusson DA, et al. Restrictive or liberal red-cell transfusion for cardiac surgery. N Eng J Med. 2017;377(22):2133-44.

20. Weisbord SD, Gallagher M, Jneid H, et al. Outcomes after angiography with sodium bicarbonate and acetylcysteine. N Eng J Med. 2018;378(7):603-14.

21. Nishimura R, Otto C, Bonow R, et al. 2017 AHA/ ACC focused update of the 2014 AHA/ACC guideline for the management of patients with valvular heart disease: a report of the American College of Cardiology/American Heart Association Task Force on Clinical Practice Guidelines. Circulation. 2018;137(15):1543-5.

22. Reardon MJ, Van Mieghem NM, Popma JJ, et al. Surgical or transcatheter aortic-valve replacement in intermediate-risk patients. $\mathrm{N}$ Eng $\mathrm{J}$ Med. 2017;376(14):1321-31.

23. Cohen DJ. Cost-effectiveness of trancatheter vs. surgical aortic valve replacement in intermediate risk patients: results from the PARTNER 2A and Sapien 3 intermediate risk trials. Presented at transcatheter therapeutics conference; October 31, 2017; Denver, Colorado.

24. Rodés-Cabau J. Ambulatory ECG monitoring with an implantable loop recorder in patients with newonset left bundle branch block following TAVR: the MARE trial. Presented at transcatheter therapeutics conference; October 31, 2017; Denver, Colorado.

25. Hafiz AM, Kalra A, Ramadan R, et al. Clinical or symptomatic leaflet thrombosis following transcatheter aortic valve replacement: insights from the US FDA MAUDE database. Struct Heart. 2017;1(5-6):256-64.

26. Kapadia SR, Kodali S, Makkar R, et al. Protection against cerebral embolism during transcatheter aortic valve replacement. J Am Coll Cardiol. 2017;69(4):367-77.

27. Mack M. Cerebral embolic protection in patients undergoing surgical aortic valve replacement (SAVR). Presented at transcatheter therapeutics conference; November 1, 2017; Denver, Colorado.

28. Gammie JS, Bartus K, Gackowski A, et al. Beatingheart mitral valve repair using a novel ePTFE cordal implantation device: prospective trial. J Am Coll Cardiol. 2018;71(1):25-36.

29. Park-Hansen J. The left atrial appendage closure by surgery study. Presented at: European Society of Cardiology congress 2017; August 28, 2017; Barcelona, Spain.

30. Reddy VY, Doshi SK, Kar S, et al. 5-year outcomes after left atrial appendage closure: from the PREVAIL and PROTECT AF trials. J Am Coll Cardiol. 2017;70(24):2964-75.

31. Søndergaard L, Kasner SE, Rhodes JF, et al. Patent foramen ovale closure or antiplatelet therapy for cryptogenic stroke. N Eng J Med. 2017;377(11):1033-42.

32. Mas JL, Derumeaux G, Guillon B, et al. Patent foramen ovale closure or anticoagulation vs. antiplatelets after stroke. N Eng J Med. 2017;377(11):1011-21.

33. Gaspardone A. Percutaneous suture-mediated patent foramen ovale (PFO) closure: early results with NobleStitch EL device. Presented at transcatheter therapeutics conference; October 30, 2017; Denver, Colorado.

34. Schauer P. Bariatric surgery versus intensive medical therapy for type 2 diabetes mellitus. STAMPEDE (surgical therapy and medications potentially eradicate diabetes efficiently). Presented at American College of Cardiology conference 2016, American Heart Association scientific sessions; New Orleans, LA, November 2016.

35. Schiavon CA, Bersch-Ferreira AC, Santucci EV, et al. Effects of bariatric surgery in obese patients with hypertension: the GATEWAY randomized trial (gastric bypass to treat obese patients with steady hypertension). Circulation. 2017;136(24):454.

36. Pujades-Rodriguez M, et al. BP TITRE: time at blood pressure target and the risk of cardiovascular diseases and mortality. Presented at American Heart Association annual scientific sessions; Anaheim, CA, Nov 2017.

37. Wright JT, Williamson JD, Whelton PK, et al. The SPRINT Research Group. A randomized trial of intensive versus standard blood-pressure control. N Engl J Med. 2015;373(22):2103-16. 
38. Johnson KC, et al. SPRINT: blood pressure measurement in the systolic blood pressure intervention trial. Presented at American Heart Association annual scientific sessions; Anaheim, CA, Nov 2017.

39. Whelton PK, Carey RM, Aronow WS, et al. ACC/ AHA/AAPA/ABC/ACPM/AGS/APhA/ASH/ASPC/ NMA/PCNA guideline for the prevention, detection, evaluation, and management of high blood pressure in adults. J Am Coll Cardiol. 2017. https:// doi.org/10.1016/j.jacc.2017.11.006.

40. Appel LJ, Moore TJ, Obarzanek E, et al. DASH Collaborative Research Group. A clinical trial of the effects of dietary patterns on blood pressure. N Engl J Med. 1997;336(16):1117-24.

41. Sacks FM, Svetkey LP, Vollmer WM, et al. DASHSodium Collaborative Research Group. Effects on blood pressure of reduced dietary sodium and the dietary approaches to stop hypertension (DASH) diet. N Engl J Med. 2001;344(1):3-10.

42. Juraschek DP, Miller ER, Weaver CM, et al. Effects of sodium reduction and the DASH diet in relation to baseline blood pressure. J Am Coll Cardiol. 2017;70(23):2849-51.

43. Bhatt DL, Kandzari DE, O'Neill WW, et al. A controlled trial of renal denervation for resistant hypertension. N Engl J Med. 2014;370(15):1393-401.

44. Kandzari DE, Bhatt DL, Brar S, et al. Predictors of blood pressure response in the SYMPLICITY HTN-3 trial. Eur Heart J. 2014;36(4):219-27.

45. Townsend RR, Mahfoud F, Kandzari DE, et al. Catheter-based renal denervation in patients with uncontrolled hypertension in the absence of antihypertensive medications (SPYRAL HTN-OFF MED): a randomised, sham-controlled, proof-of-concept trial. Lancet. 2017;390:2160-70.

46. Marso AP, Daniels G, et al. Liraglutide and cardiovascular outcomes in type 2 diabetes. $\mathrm{N}$ Eng $\mathrm{J}$ Med. 2016;375:311-22.

47. Mentz R. EXSCEL: effect of exenatide once-weekly on clinical outcomes in patients with type 2 diabetes mellitus and cardiovascular disease: insights from the EXSCEL trial. Presented at American Heart Association annual scientific sessions; Denver, CO, Nov 2017.

48. Zinman B, Wanner C, Lachin JM, et al. Empagliflozin, cardiovascular outcomes, and mortality in type 2 diabetes. $\mathrm{N}$ Engl J Med. 2015;373(22):2117-28.

49. Verma S, Mazer CD, Al-Omran M, et al. Cardiovascular outcomes and safety of empagliflozin in patients with type 2 diabetes mellitus and peripheral artery disease: a subanalysis of EMPA-REG OUTCOME. Circulation. 2018;137(4):405-7.

50. Mahaffey KW, Neal B, Perkovic V. Canagliflozin for primary and secondary prevention of cardiovascular events: results from the CANVAS program (canagliflozin cardiovascular assessment study). Circulation. 2018;137(4):323-34.

51. Neal B, Perkovic V, Mahaffey KW, et al. Canagliflozin and cardiovascular and renal events in type 2 diabetes. N Engl J Med. 2017;377:644-57.

52. Sabatine MC, Giugliano RP, Keech AC, et al. Evolocumab and clinical outcomes in patients with cardiovascular disease. $\mathrm{N}$ Engl $\mathrm{J}$ Med. 2017;376:1713-22.

53. Bonaca MP, Nault P, Giugliano RP, et al. Low-density lipoprotein cholesterol lowering with evolocumab and outcomes in patients with peripheral artery disease: insights from the FOURIER trial (further cardiovascular outcomes research with PCSK9 inhibition in subjects with elevated risk). Circulation. 2018;137(4):338-50.

54. Sabatine MC, et al. Clinical benefit of evolocumab in patients with a history of MI: an analysis from FOURIER. Presented at American Heart Association annual scientific sessions; Anaheim, CA, Nov 2017.

55. Lipinski MJ, Benedetto U, Escarcega RO, et al. The impact of proprotein convertase subtilisin-kexin type 9 serine protease inhibitors on lipid levels and outcomes in patients with primary hypercholesterolaemia: a network meta-analysis. Eur Heart J. 2016;37(6):536-45.

56. Giugliano RP, Mach F, Zavitz K, et al. Cognitive function in a randomized trial of evolocumab. N Engl J Med. 2017;377(7):633-43.

57. Ridker PM, Revkin J, Amarenco P, et al. Cardiovascular efficacy and safety of bococizumab in highrisk patients. N Engl J Med. 2017;376(16):1527-39.

58. Fitzgerald K, White S, Borodovsky A, et al. A highly durable RNAi therapeutic inhibitor of PCSK9. N Engl J Med. 2017;376(1):41-51.

59. Ray KK, Landmesser U, Leiter LA, et al. Inclisiran in patients at high cardiovascular risk with elevated LDL cholesterol. N Engl J Med. 2017;376(15):1430-40.

60. Ray KK, et al. Impact of a single or two dose regimen of inclisiran, a novel siRNA inhibitor to PCSK9 on time averaged reductions in LDL-C over 1 year. ORION 1. Presented at European Society of Cardiology annual scientific sessions; Bareclona, Aug 2017. 
61. HPS3/TIMI55-REVEAL Collaborative Group, Bowman L, Hopewell JC, et al. Effects of anacetrapib in patients with atherosclerotic vascular disease. N Engl J Med. 2017;377(13):1217-27.

62. Bowman L, et al. REVEAL: effects of anacetrapib on the incidence of new-onset diabetes mellitus and on vascular events in people with diabetes. Presented at American Heart Association annual scientific sessions; Anaheim, CA, Nov 2017.

63. Tardif JC, Ballantyne CM, Barter P, et al. Effects of the high-density lipoprotein mimetic agent CER001 on coronary atherosclerosis in patients with acute coronary syndromes: a randomized trial. Eur Heart J. 2014;35(46):3277-86.

64. Nicholls S, et al. CARAT: effect of serial infusions of CER-001, a pre-beta high-density lipoprotein mimetic on coronary atherosclerosis: results of the CARAT study. Presented at American College of Cardiology annual scientific sessions; Washington, DC, March 2017.

65. Montalescot G, Sechtem U, Achenbach S, et al. 2013 ESC guidelines on the management of stable coronary artery disease: the Task Force on the management of stable coronary artery disease of the European Society of Cardiology. Eur Heart J. 2013;34:2949-3003.

66. Kimura T, et al. REAL-CAD: does high-intensity pitavastatin therapy further improve clinical outcomes? The REAL-CAD study in 13,054 patients with stable coronary artery disease. Presented at American Heart Association annual scientific sessions; Anaheim, CA, Nov 2017.

67. Ridker PM, Hennekens $\mathrm{CH}$, Buring JE, Rifai N. C-reactive protein and other markers of inflammation in the prediction of cardiovascular disease in women. N Engl J Med. 2000;342(12):836-43.

68. Ridker PM, Everett BM, Thuren T, et al. Antiinflammatory therapy with canakinumab for atherosclerotic disease. $\mathrm{N}$ Engl $\mathrm{J}$ Med. 2017;377(12):1119-31.

69. Ridker PM, MacFadyen JG, Everett BM, et al. Relationship of C-reactive protein reduction to cardiovascular event reduction following treatment with canakinumab: a secondary analysis from the CANTOS randomised controlled trial. Lancet. 2017;391(10118):319-28.

70. Dehghan M, Mente A, Zhang X, et al. Associations of fats and carbohydrate intake with cardiovascular disease and mortality in 18 countries from five continents (PURE): a prospective cohort study. Lancet. 2017;390(10107):2050-62.
71. Ibanez B, James S, Agewall S, et al. 2017 ESC guidelines for the management of acute myocardial infarction in patients presenting with ST-segment elevation: The Task Force for the management of acute myocardial infarction in patients presenting with ST-segment elevation of the European Society of Cardiology (ESC). Eur Heart J. 2018;39(2):119-77.

72. Stub D, Smith K, Bernard S, et al. Air versus oxygen in ST-segment-elevation myocardial infarction. Circulation. 2015;131(24):2143-50.

73. Hofmann R, James SK, Jernberg T. Oxygen therapy in suspected acute myocardial infarction. N Engl J Med. 2017;377(13):1240-9.

74. Kedhi E. Six versus twelve months of dual antiplatelet therapy after drug-eluting stent implantation in st-elevation myocardial infarction-primary results from the DAPT-STEMI trial. Presented at transcatheter cardiovascular therapeutics; Denver, CO, 2017.

75. Omerovic E, et al. Pretreatment with P2Y12 receptor antagonists is not associated with improved clinical outcomes in STEMI: a report from SCAAR. Presented at European Society of Cardiology annual scientific sessions; Barcelona, Aug 2017.

76. Montalescot G, van't Hof AW, Lapostolle F, et al. Prehospital ticagrelor in ST-segment elevation myocardial infarction. $\mathrm{N}$ Engl $\mathrm{J}$ Med. 2014;371(24):1016-27.

77. Cayla G, Cuisset T, Silvain J, et al. Platelet function monitoring to adjust antiplatelet therapy in elderly patients stented for an acute coronary syndrome (ANTARTIC): an open label, blinded endpoint, randomised controlled superiority trial. Lancet. 2016;388(10055):2015-21.

78. Sibbing D, Aradi D, Jacobshagen C, et al. Guided deescalation of antiplatelet treatment in patients with acute coronary syndrome undergoing percutaneous coronary intervention (TROPICAL-ACS): a randomised, open-label, multicentre trial. Lancet. 2017;390(10104):1747-57.

79. Motovska Z, Hlinomaz O, Kala P, et al. 1-year outcomes of patients undergoing primary angioplasty for myocardial infarction treated with prasugrel versus ticagrelor. J Am Coll Cardiol. 2018;71(4):371-81.

80. Shahzad A, Kemp I, Mars C, et al. Unfractionated heparin versus bivalirudin in primary percutaneous coronary intervention (HEAT-PPCI): an open-label, single centre, randomised controlled trial. Lancet. 2014;384(9957):1849-58.

81. Han Y, Guo J, Zheng Y, et al. Bivalirudin vs heparin with or without tirofiban during primary 
percutaneous coronary intervention in acute myocardial infarction: the BRIGHT randomized clinical trial. JAMA. 2015;313(13):1336-46.

82. Schulz S, Richardt G, Laugwitz KL, et al. Prasugrel plus bivalirudin vs. clopidogrel plus heparin in patients with ST-segment elevation myocardial infarction. Eur Heart J. 2014;35(34):2285-94.

83. Erlinge D, Omerovic E, Fröbert O, et al. Bivalirudin versus heparin monotherapy in myocardial infarction. N Engl J Med. 2017;377(12):1132-42.

84. Mega JL, Braunwald E, Wiviott SD, et al. Rivaroxaban in patients with a recent acute coronary syndrome. N Engl J Med. 2012;366(1):9-19.

85. Ohman EM, Roe MT, Steg PG, et al. Clinically significant bleeding with low-dose rivaroxaban versus aspirin, in addition to P2Y12 inhibition, in acute coronary syndromes (GEMINI-ACS-1): a doubleblind, multicentre, randomised trial. Lancet. 2017;389(10081):1799-808.

86. Bonaca MP, Bhatt DL, Cohen M, et al. Long-term use of ticagrelor in patients with prior myocardial infarction. N Engl J Med. 2015;372(10081):1791-800.

87. Alexander JH, Lopes RD, James S, et al. Apixaban with antiplatelet therapy after acute coronary syndrome. NEngl J Med. 2011;365(8):699-708.

88. Connolly SJ, Eikelboom JW, Bosch J, et al. Rivaroxaban with or without aspirin in patients with stable coronary artery disease: an international, randomized, double-blind, placebo-controlled trial. Lancet. 2017. https://doi.org/10.1016/ S0140-6736(17)32458-3.

89. Anand SS, Bosch J, Connolly SJ, et al. Rivaroxaban with or without aspirin in patients with stable peripheral or carotid artery disease: an international, randomized, double-blind, placebo-controlled trial. Lancet. 2017. https://doi.org/10.1016/S01406736(17)32.

90. Gibson CM, Mehran R, Bode C, et al. Prevention of bleeding in patients with atrial fibrillation undergoing PCI. N Engl J Med. 2016;375:2423-34.

91. Cannon CP, Bhatt DL, Oldgren J, et al. Dual antithrombotic therapy with dabigatran after PCI in atrial fibrillation. $\mathrm{N}$ Engl J Med. 2017;377(16):1513-24.

92. Oldgren J, et al. RE-DUAL PCI: subgroup analysis from the RE-DUAL PCI trial: dual antithrombotic therapy with dabigatran in patients with atrial fibrillation undergoing percutaneous coronary intervention. Presented at American Heart Association annual scientific sessions; Anaheim, CA, Nov 2017.
93. Zhao Q, et al. Efficacy of different antiplatelet therapy strategy after coronary artery bypass grafting (DACAB)(NCT). Presented at American Heart Association annual scientific sessions; Anaheim, CA, Nov 2017.

94. Graham M. POISE 2: PCI patients may benefit from perioperative aspirin. Presented at American Heart Association scientific sessions; Anaheim, CA, 2017.

95. Hemmelgarn B. Comparative safety of direct oral anticoagulants and warfarin in venous thromboembolism: multicentre, population based observational study; presented at TCT 2017. Original DATA-Jun $M$ et al. Comparative safety of direct oral anticoagulants and warfarin in venous thromboembolism: multicentre, population based observational study. BMJ. 2017;359:j4323.

96. Weitz J, Lensing A, Prins M, et al. Rivaroxaban or Aspirin for extended treatment of venous thromboembolism. N Eng J Med. 2017;376(13):1211-22.

97. Agnelli G, Buller HR, Cohen A, et al. Apixaban for extended treatment of venous thromboembolism. N Eng J Med. 2013;368(8):699-708.

98. Win-Kuang S, Sheldon RS, Benditt D, et al. 2017 ACC/AHA/HRS guideline for the evaluation and management of patients with syncope. Circulation. 2017;136(5):e25-59.

99. Baron-Esquivias G, et al. Closed loop stimulation for neuromediated syncope SPAIN study (NCT01621464)-late-breaking clinical trials. Presented at American College of Cardiology scientific session; March 19, 2017; Washington, DC

100. Halcox JPJ, Wareham K, Cardew A, et al. Assessment of remote heart rhythm sampling using the AliveCor heart monitor to screen for atrial fibrillation. The REHEARSE-AF study. Circulation. 2017;136(19):1784-94.

101. Vinereanu D, Lopes RD, Bahit M, et al. A multifaceted intervention to improve treatment with oral anticoagulants in atrial fibrillation: an international, cluster randomised trial-IMPACT AF. Lancet. 2017;390(10104):1737-46.

102. Ezelowitz M. Apixaban versus conventional therapy in anticoagulation naïve patients with atrial fibrillation undergoing cardioversion: EMANATE trial. Presented at European Society of Cardiology Congress; Barcelona, August 28, 2017.

103. Cappato R, Ezekowitz MD, Klein AL, et al. Explore the efficacy and safety of once-daily oral rivaroxaban for the prevention of cardiovascular events in subjects with nonvalvular atrial fibrillation scheduled for cardioversion (X-VERT). Eur Heart J. 2014;35(47):3346-55. 
104. Goette A, Merino JL, Ezekowitz MD, et al. Edoxaban vs. warfarin in subjects undergoing cardioversion of atrial fibrillation (ENSURE-AF). Lancet. 2016;388(10055):1995-2003.

105. Bavry A, et al. Routine vs. aggressive rhythm control for prevention of early persistent atrial fibrillation in heart failure study-RACE 3. Presented at European Society of Cardiology congress; Barcelona, August 28, 2017.

106. Bavry A, et al. Catheter ablation compared with pharmacological therapy for atrial fibrillationCAPTAF. Presented at European Society of Cardiology congress; Barcelona, August 28, 2017.

107. Marrouche NF, Brachmann J, Andresen D, et al. Catheter ablation for atrial fibrillation with heart failure. N Eng J Med. 2018;378(5):417-27.

108. Prabhu S, Taylor AJ, Costello BT, et al. Catheter ablation versus medical rate control in atrial fibrillation and systolic dysfunction-CAMERA MRI (ACTRN 12613000880741). J Am Coll Cardiol. 2017;70(16):1949-61.

109. Birnie DH, Healey JS, Wells GA, et al. Pacemaker or defibrillator surgery without interruption of anticoagulation (BRUISE-CONTROL 1). N Eng J Med. 2013;368(22):2084-93.

110. Birnie DH. Strategy of continued versus interrupted novel oral anti-coagulant at time of device surgery in patients with moderate to high risk of arterial thromboembolic events (BRUISECONTROL2). Presented at American Heart Association annual scientific sessions; Anaheim, CA, Nov 2017.

111. Nogami A. Ablation perioperative dabigatran in use envisioning in Japan-ABRIDGE-J. Presented at American Heart Association annual scientific sessions; Anaheim, CA, Nov 2017.

112. Mehta RH, Leimberger JD, van Diepen S, et al. Levosimendan in patients with left ventricular dysfunction undergoing cardiac surgery (LEVO-CTS) (NCT 02025621). N Eng J Med. 2017;376(21):2032-42.

113. Nijhawan N, Nicolosi AC, Montgomery MW, et al. Levosimendan enhances cardiac performance after cardiopulmonary bypass: a prospective, randomized, placebo-controlled trial. J Cardiovasc Pharmacol. 1999;34(2):219-28.

114. https://www.doc2do.com/hcm/webHCM.html. Accessed 13 June 2018.

115. O'Mahony C. An international external validation study of the 2014 European Society of Cardiology guideline on sudden cardiac death in hypertrophic cardiomyopathy, HCM-EVIDENCE study. Presented at European Society of Cardiology congress; Barcelona, August 29, 2017.

116. Pitt B, Remme W, Zannard F, et al. Eplerenone, a selective aldosterone blocker, in patients with left ventricular dysfunction after myocardial infarction. N Eng J Med. 2003;348(14):1309-21.

117. Beygui F, Cayla G, Roule V, et al. Early aldosterone blockade in acute myocardial infarction: the ALBATROSS randomised clinical trial. J Am Coll Cardiol. 2016;67(16):1917-27.

118. Montalescot G, Pitt B, Lopez de Sa E, et al. Early eplerenone treatment in patients with acute ST-elevation myocardial infarction without heart failure: the randomised double blind reminder study. Eur Heart J. 2014;35(34):2295-302.

119. Allen L, et al. Trial of a decision support intervention for patients and caregivers offered destination therapy heart assist device (DECIDE-LVAD). Presented at American Heart Association annual scientific sessions; Anaheim,CA, Nov 2017.

120. Feldman T, Mauri L, Kahwash R, et al. A transcatheter interatrial shunt device for the treatment of heart failure with preserved ejection fraction (REDUCE LAP HF I): a phase 2, randomized, shamcontrolled trial. Circulation. 2017;137(4):364-75.

121. Henry TD. Allogenic Stem cell to achieve myocardial regeneration (ALLSTAR). Presented at American Heart Association annual scientific sessions; Anaheim, CA, Nov 2017.

122. Victor RG, et al. Halt cardiomyopathy progression in Duchenne (HOPE-Duchenne). Presented at American Heart Association annual scientific sessions; Anaheim, CA, Nov 2017. 